\title{
MEZCLAS BINARIAS DE LÍOUIDO IÓNICO CON SOLVENTE MOLECULAR: PREDICCIÓN DEL VOLUMEN DE EXCESO MOLAR DESDE ÍNDICES DE REFRACCIÓN
}

\author{
BINARY MIXTURES OF IONIC LIOUID WITH MOLECULAR SOLVENT: PREDICTION OF EXCESS \\ MOLAR VOLUMES FROM REFRACTIVE INDEX \\ Marlon Doney Martínez Reina \\ Ingeniero de Materiales, Estudiante de Doctorado Ciencias Químicas. \\ Departamento de Química, Universidad del Valle, Cali, Colombia. \\ marlon.martinez@correounivalle.edu.co \\ Eliseo Amado González \\ Licenciado en Química y Biología, Magister en Ciencias Químicas, Doctor en Ciencias Químicas. Profesor Asociado \\ Facultad de Ciencias Básicas, Universidad de Pamplona, Pamplona, Colombia. \\ eamadogon@gmail.com
}

Fecha de recepción: 29 de junio de 2011

Fecha de aprobación: 19 de diciembre de 2011

\section{RESUMEN}

El volumen de exceso molar de 30 mezclas binarias que contienen líquidos iónicos de imidazolio con diferentes solventes moleculares: (metanol, etanol, 1-propanol, 2-propanol, acetona, 2butanona, 2-pentanona, acetato de metilo, acetato de etilo, acetato de butilo, carbonato de dimetilo, carbonato de dietilo, nitrometano, 1,3-dicloropropano y etilenglicoll,se predice desde datos de índice de refracción, usando tres tipos de métodos acoplados con diferentes reglas de mezcla para el cálculo del índice de refracción: Lorentz-Lorenz, Dale-Gladstone, Eykman, AragoBiot, Newton y Oster. En estos sistemas, las interacciones moleculares y las fuerzas intermoleculares provocan, durante la mezcla, desviaciones positivas o negativas del comportamiento ideal. Los resultados obtenidos se analizan en términos de la naturaleza del líquido iónico y solvente molecular.

Palabras clave: volumen de exceso molar, índice de refracción, predicción, líquido iónico, solvente molecular.

\begin{abstract}
The excess molar volumes of 30 binary mixtures containing ionic liquids of imidazolium with different molecular solvents: (methanol, ethanol, propan-1-ol, propan-2-ol, acetone, 2butanone, 2-pentanone, methyl acetate, ethyl acetate, butyl acetate, dimethyl carbonate,
\end{abstract}


diethyl carbonate, nitromethane, 1,3-dichloropropane and ethylene glycol), were predicted from the refractive index data, using three types of methods coupled with several different mixing rules for refractive index calculations: Lorentz-Lorenz, Dale-Gladstone, Eykman, Arago-Biot, Newton and Oster. In these systems, molecular interactions and intermolecular forces during mixing produce positive or negative deviations from ideal behavior. The results are analyzed in terms of the nature of ionic liquid and molecular solvent.

Keywords: excess molar volume, refractive index, prediction, liquid ionic, molecular solvent.

\section{INTRODUCCIÓN}

El término líquido iónico se ha utilizado en los últimos años, para definir una familia de compuestos constituida por iones que tienen un punto de fusión inferior a 373.15 K [1] y [2]. Son sales orgánicas con iones muy asimétricos y voluminosos que presentan fuerzas atractivas más débiles que las sales iónicas convencionales, con un alto grado de asimetría que inhibe su cristalización. En su estructura, presentan un catión orgánico con un heteroátomo (N, P o S): imidazolio, piridinio, pirrolidinio, alquil amonio, fosfonio y sulfonio asociado a un anión orgánico o inorgánico: alquil sulfato, haluro, nitrato, acetato, tetrafluoroborato, hexafluorofosfato y otros [3].

El interés por usar líquidos iónicos y disolventes en diferentes procesos químicos, se debe a que estos compuestos son líquidos en un amplio intervalo de temperatura, presentan una buena estabilidad térmica, tienen una elevada polaridad, permanecen inalterados cuando se mezclan con diferentes compuestos orgánicos, son miscibles/inmiscibles con sustancias orgánicas o inorgánicas y lo más importante, tienen una presión de vapor despreciable [4] y [5], que hace que en sus aplicaciones, no emitan compuestos orgánicos volátiles (COVs), y se estudien como potenciales solventes verdes para reemplazar los solventes orgánicos volátiles tradicionales en procesos de catálisis, síntesis orgánica, técnicas de separación, electroquímica, química analítica e investigaciones en nano materiales [6], [7], [8], [9], [10] y [11].

Estas aplicaciones requieren de datos fisicoquímicos de líquidos iónicos y sus mezclas con otros componentes. En la literatura, se han reportado propiedades termodinámicas y termofísicas: densidad, viscosidad, índices de refracción, velocidad del sonido, tensión superficial, conductividad eléctrica, solubilidad y equilibrio de fases de mezclas binarias entre un líquido iónico y un solvente molecular [12], [13], [14], [15], [16] y [17].

El estudio de propiedades volumétricas de sistemas binarios con líquidos iónicos, tiene dos objetivos: proporcionar datos de densidad de mezcla, necesarios en cálculos de transferencia de masa, y comprender las interacciones soluto-solvente entre líquidos iónicos y solventes moleculares. Estas interacciones se estudian en términos de propiedades de mezcla como el volumen de exceso molar $\left(V_{M}^{E}\right)$, que para una mezcla binaria, se define así [18]: 


$$
V_{M}^{E}=\sum_{i=1}^{2} x_{i} M_{i}\left(\rho^{-1}-\rho_{i}^{o-1}\right)
$$

donde, $\rho$ es la densidad de la mezcla, $\rho_{i}^{o}, M_{i}$ y $x_{i}$ es la densidad, peso molecular y fracción molar de los componentes 1 y 2 del sistema binario. La ecuación (1) indica que para calcular el volumen de exceso molar en un sistema binario, se requiere determinar en forma experimental la densidad de la mezcla en todo el rango de fracción molar. Las técnicas experimentales para determinar el índice de refracción de sistemas líquidos son relativamente más simples [19]. Aplicando la regla de mezclas, es posible predecir el volumen de exceso molar desde índices de refracción de sistemas multicomponentes [20] y [21].

Para predecir el $V_{M}^{E}$ desde índices de refracción de mezcla $n_{D}$, se consideran tres métodos [19]:

Método I. Ecuación propuesta por Nakata y Sakurai en 1987 [21], basada en el uso de la refracción específica, $f\left(n_{D}\right) / \rho$, y la definición de volumen de exceso molar:

$$
\frac{f\left(n_{D}\right)}{\rho}=\sum_{i=1}^{2} w_{i} \frac{f\left(n_{D i}\right)}{\rho_{i}}
$$

Despejando densidad de la mezcla en la ecuación (2) y reemplazando en la ecuación (1), se obtiene la siguiente expresión para el volumen de exceso molar:

$$
V_{M}^{E}=\sum_{i=1}^{2}\left[\left(f\left(n_{D i}\right)-f\left(n_{D}\right)\right)\left(\frac{x_{i} V_{M i}}{f\left(n_{D}\right)}\right)\right]
$$

Donde $w_{i}, \rho_{i}, n_{D i}$ y $x_{i}$ son fracción másica, densidad, índice de refracción y fracción molar del componente $i, f\left(n_{D i}\right)$ y $f\left(n_{D}\right)$ representan funciones del índice de refracción de los componentes puros y de la mezcla; y $V_{M i}$ es el volumen molar pre-mezcla del componente $i$.

Típicas funciones $f\left(n_{D}\right)$ son Lorentz-Lorenz $\left(\mathrm{L}-\mathrm{L},\left(n_{D}^{2}-1\right) /\left(n_{D}^{2}+2\right)\right)$. Dale-Gladstone (D-G, $\left.n_{D}-1\right)$, Eykman $\left(\right.$ Eyk, $\left.\left(n_{D}^{2}-1\right) /\left(n_{D}+0.4\right)\right)$, Arago-Biot $\left(\mathrm{A}-\mathrm{B}, n_{D}\right)$. Newton (New, $\left.n_{D}^{2}-1\right) \mathrm{y}$ Oster (OS, $\left.\left(n_{D}^{2}-1\right)\left(2 n_{D}^{2}+1\right) / n_{D}^{2}\right)[22]$.

Método II. Considerando $V_{M}^{E}$ en la ecuación (3), como una función de $n_{D}$ y expandiendo hasta primer orden [19] con $n_{D \phi}=n_{D 1} \phi_{1}+n_{D 2} \phi_{2}$ se obtiene:

$$
V_{M}^{E}=\sum_{i=1}^{2} x_{i} V_{M i}\left(\frac{f\left(n_{D i}\right)}{f\left(n_{D \phi}\right)}-1\right)-\Delta_{\phi} n_{D}\left[\left(\frac{f^{\prime}\left(n_{D \phi}\right)}{f\left(n_{D \phi}\right)^{2}}\right) \sum_{i=1}^{2} x_{i} V_{M i} f\left(n_{D i}\right)\right]
$$


Donde $f\left(n_{D \phi}\right)$, representa una función del índice de refracción de una mezcla como en el método I; $f^{\prime}\left(n_{D \phi}\right)$, es la primera derivada de $f\left(n_{D \phi}\right)$ y $\Delta_{\phi} n_{D}$ representa el cambio del índice de refracción de la mezcla binaria desde la mezcla ideal:

$$
\Delta_{\phi} n_{D}=n_{D}-\left(n_{D 1} \phi_{1}+n_{D 2} \phi_{2}\right)
$$

Donde $n_{D}$ es el índice de refracción de la mezcla, $n_{D 1}$ y $n_{D 2}$ son los índices de refracción de los componentes puros y $\phi_{1}$ y $\phi_{2}$ es la fracción en volumen basada en el volumen molar de los componentes puros.

Método III. Para mezclas isorrefractivas $n_{D 1} \simeq n_{D 2}$ la ecuación (4) se reduce a [19]:

$$
V_{M}^{E}=-\Delta_{\phi} n_{D}\left[\left(\frac{f^{\prime}\left(n_{D 1}\right)}{f\left(n_{D 1}\right)}\right) \sum_{i=1}^{2} x_{i} V_{M i}\right]
$$

Donde $f\left(n_{D 1}\right)$, representa la función del índice de refracción como en el método I y $f^{\prime}\left(n_{D 1}\right)$ la primera derivada de la función L-L, D-G, E y k, A-B, New y Os evaluadas en el índice de refracción isorrefractivo; es decir, el índice de refracción del líquido iónico puro.

\section{MEZCLAS BINARIAS}

La predicción del $V_{M}^{E}$ a $298.15 \mathrm{~K}$ desde datos de índice de refracción fue realizada en 30 mezclas binarias: líquido iónico de imidazolio-solvente molecular con datos tomados de la literatura; en la Figura 1, se muestra la estructura de los líquidos iónicos considerados en las siguientes mezclas:

Grupo 1. Mezclas binarias líquido iónico de imidazolio-solvente molecular (efecto de longitud

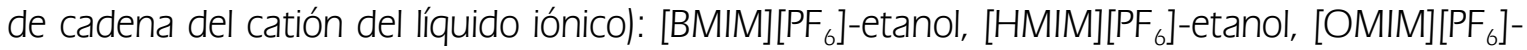
etanol, $[\mathrm{BMIM}]\left[\mathrm{PF}_{6}\right]-2$-butanona, [HMIM] $\left[\mathrm{PF}_{6}\right]-2$-butanona, [OMIM][PF$\left.F_{6}\right]$-2-butanona, $[\mathrm{BMIM}]\left[\mathrm{PF}_{6}\right]$-acetato de etilo, $[\mathrm{HMIM}]\left[\mathrm{PF}_{6}\right]$-acetato de etilo y $[\mathrm{OMIM}]\left[\mathrm{PF}_{6}\right]$-acetato de etilo.

Grupo 2. Mezclas binarias líquido iónico de imidazolio-alcohol lefecto de longitud de cadena del alcohol): [EMIM] [EtSO $\left.{ }_{4}\right]$-metanol, [EMIM] $\left[\mathrm{EtSO}_{4}\right]$-etanol, [EMIM][EtSO $]$-1-propanol y $[\mathrm{EMIM}]\left[\mathrm{EtSO}_{4}\right]$-2-propanol.

Grupo 3. Mezclas binarias líquido iónico de imidazolio-etanol (efecto del tipo de anión del

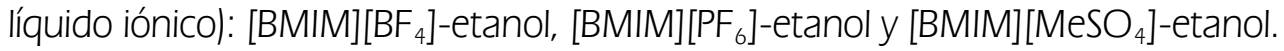



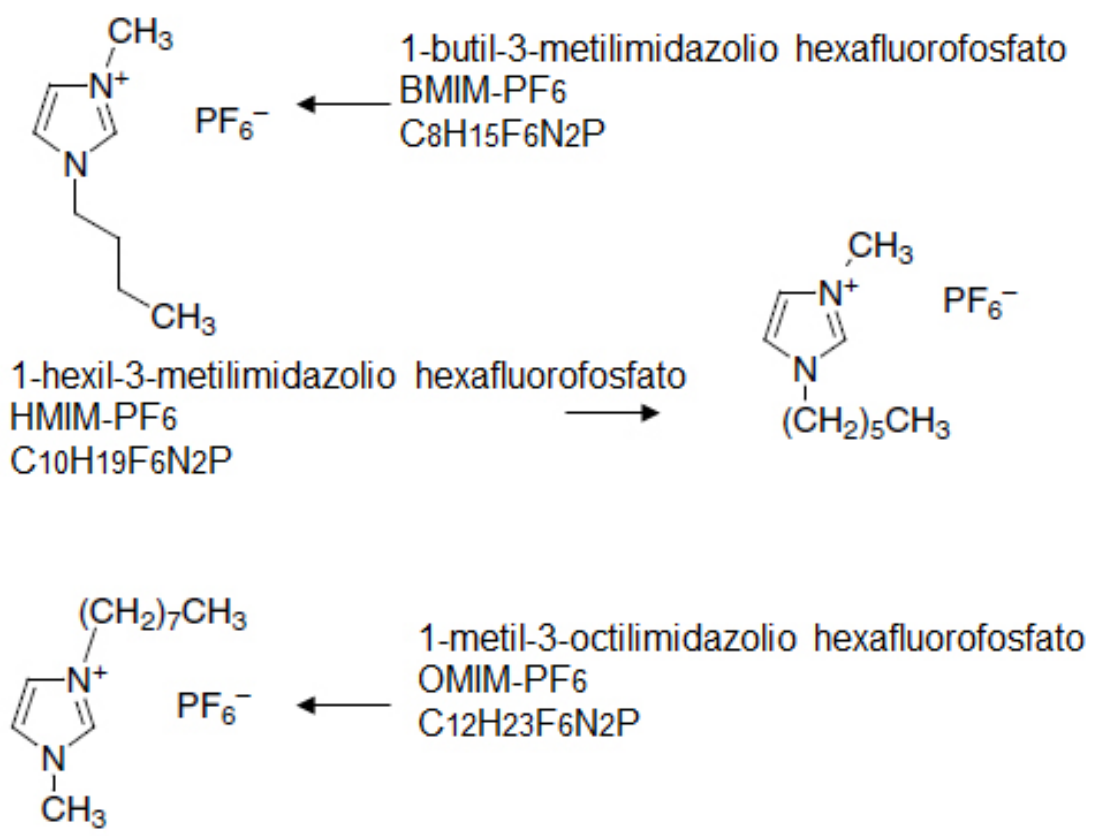

1-etil-3-metilimidazolio etilsulfato EMIM-EtSO4<smiles>CCOS(=O)(=O)[O-]</smiles>

$\mathrm{C} 8 \mathrm{H} 16 \mathrm{~N} 2 \mathrm{O} 4 \mathrm{~S}$

1-butil-3-metilimidazolio tetrafluoroborato<smiles>CCCCn1cc[n+](C)c1</smiles>

BMIM-BF4

$\mathrm{C}_{8} \mathrm{H}_{15} \mathrm{BF} 4 \mathrm{~N} 2$

1-Butil-3-metilimidazolio metilsulfato BMIM-MeSO4

$\mathrm{C} 9 \mathrm{H}_{18} \mathrm{~N}_{2} \mathrm{O} 4 \mathrm{~S}$<smiles>CCCCn1cc[n+](C)c1</smiles><smiles>COS(=O)(=O)[O-]</smiles>

1,3-dimetilimidazolio metilsulfato MMIM-MeSO4 $\mathrm{C}_{6} \mathrm{H}_{12} \mathrm{~N} 2 \mathrm{O} 4 \mathrm{~S}$

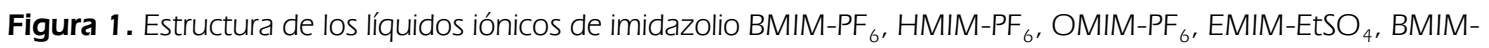
$\mathrm{BF}_{4},{\mathrm{BMIM}-\mathrm{MeSO}_{4} \text { y MMIM-MeSO}}_{4}$ 
Grupo 4. Mezclas binarias líquido iónico de imidazolio-éster (efecto de la naturaleza del éster): $[\mathrm{HMIM}]\left[\mathrm{PF}_{6}\right]$-acetato de metilo, $[\mathrm{HMIM}]\left[\mathrm{PF}_{6}\right]$-acetato de etilo, $[\mathrm{HMIM}]\left[\mathrm{PF}_{6}\right]$-acetato de butilo, $[\mathrm{HMIM}]\left[\mathrm{PF}_{6}\right]$-carbonato de dimetilo (DMC) y $\left[\mathrm{HMIM}\left[\mathrm{PF}_{6}\right]\right.$-carbonato de dietilo (DEC).

Grupo 5. Mezclas binarias líquido iónico de imidazolio-cetona (efecto de longitud de cadena de la cetona): $[\mathrm{HMIM}]\left[\mathrm{PF}_{6}\right]$-acetona, $[\mathrm{HMIM}]\left[\mathrm{PF}_{6}\right]$-2-butanona y $[\mathrm{HMIM}]\left[\mathrm{PF}_{6}\right]$-2-pentanona.

Grupo 6. Mezclas binarias líquido iónico de imidazolio - solvente molecular (efecto de la

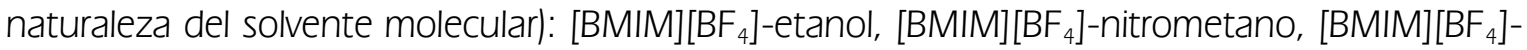

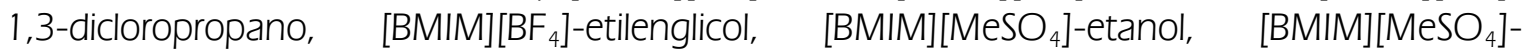
nitrometano, [BMIM][MeSO 4 ]-1,3-dicloropropano, [MMIM] $\left[\mathrm{MeSO}_{4}\right]$-etanol, [MMIM][MeSO$\left.{ }_{4}\right]$-2butanona, [MMIM][MeSO $\left.{ }_{4}\right]$-2-propanol y [MMIM][MeSO$\left.{ }_{4}\right]$-acetato de etilo.

En la Tabla 1, se reportan a $298.15 \mathrm{~K}$, los valores de densidad $\left(\rho_{i}\right)$, índice de refracción $\left(n_{D i}\right)$ y volumen molar $\left(V_{M i}\right)$ de los líquidos iónicos y solventes moleculares considerados en este estudio.

Tabla 1. Densidad en $\left(\mathrm{g} \mathrm{cm}^{-3}\right)$, índice de refracción y volumen molar en $\left(\mathrm{cm}^{3} \mathrm{~mol}^{-1}\right)$ de los componentes puros a $298.15 \mathrm{~K}$

\begin{tabular}{|c|c|c|c|c|c|c|c|}
\hline $\begin{array}{l}\text { Componente } \\
\text { [Referencia] }\end{array}$ & $\rho_{i}$ & $n_{D i}$ & $V_{M i}$ & $\begin{array}{l}\text { Componente } \\
\text { [Referencia] }\end{array}$ & $\rho_{i}$ & $n_{D i}$ & $V_{M i}$ \\
\hline $\begin{array}{c}{[\mathrm{BMIM}]\left[\mathrm{PF}_{6}\right]} \\
{[23]}\end{array}$ & 1.3673 & 1.4094 & 207.84 & $\begin{array}{c}\text { acetona } \\
{[25]}\end{array}$ & 0.7844 & 1.3557 & 78.04 \\
\hline $\begin{array}{c}{[\mathrm{MMIM}]\left[\mathrm{MeSO}_{4}\right]} \\
{[23]}\end{array}$ & 1.3272 & 1.4827 & 156.90 & $\begin{array}{c}\text { 2-pentanona } \\
{[25]} \\
\end{array}$ & 0.8014 & 1.3832 & 107.48 \\
\hline $\begin{array}{c}{[\mathrm{HMIM}]\left[\mathrm{PF}_{6}\right]} \\
{[23]}\end{array}$ & 1.2937 & 1.4179 & 241.35 & $\begin{array}{c}\text { 2-butanona } \\
{[26]}\end{array}$ & 0.7997 & 1.3762 & 90.17 \\
\hline $\begin{array}{c}{[\mathrm{OMIM}]\left[\mathrm{PF}_{6}\right]} \\
{[23]}\end{array}$ & 1.2357 & 1.4230 & 275.39 & $\begin{array}{c}\text { metanol } \\
\text { [27] }\end{array}$ & 0.7864 & 1.3269 & 40.75 \\
\hline $\begin{array}{c}{[\mathrm{BMIM}]\left[\mathrm{MeSO}_{4}\right]} \\
{[23]}\end{array}$ & 1.2122 & 1.4794 & 206.50 & $\begin{array}{c}\text { 1-propanol } \\
\text { [27] }\end{array}$ & 0.7996 & 1.3831 & 75.16 \\
\hline $\begin{array}{c}\text { etanol } \\
{[23]}\end{array}$ & 0.7851 & 1.3593 & 58.68 & $\begin{array}{c}\text { 2-propanol } \\
{[27]}\end{array}$ & 0.7807 & 1.3750 & 76.98 \\
\hline $\begin{array}{c}\text { acetato de etilo } \\
\text { [24] }\end{array}$ & 0.8944 & 1.3698 & 98.51 & $\begin{array}{c}{[\mathrm{EMIM}]\left[\mathrm{EtSO}_{4}\right]} \\
{[28]}\end{array}$ & 1.2376 & 1.4794 & 190.92 \\
\hline $\begin{array}{c}\text { acetato de metilo } \\
{[25]}\end{array}$ & 0.9269 & 1.3588 & 79.92 & $\begin{array}{c}{\left[{\mathrm{BMIM}]\left[\mathrm{BF}_{4}\right]}_{[29]}\right.} \\
\end{array}$ & 1.2014 & 1.4219 & 188.13 \\
\hline $\begin{array}{c}\text { acetato de butilo } \\
\end{array}$ & 0.8763 & 1.3919 & 132.56 & $\begin{array}{c}\text { nitrometano } \\
\text { [29] }\end{array}$ & 1.1312 & 1.3796 & 53.96 \\
\hline $\begin{array}{c}\mathrm{DMC} \\
{[25]}\end{array}$ & 1.0633 & 1.3664 & 84.72 & $\begin{array}{c}\text { 1,3- } \\
\text { dicloropropano } \\
{[29]}\end{array}$ & 1.1719 & 1.4460 & 95.83 \\
\hline $\begin{array}{l}\text { DEC } \\
{[25]}\end{array}$ & 0.9692 & 1.3828 & 121.89 & $\begin{array}{c}\text { etlienglicol } \\
\text { [29] }\end{array}$ & 1.1100 & 1.4304 & 55.92 \\
\hline
\end{tabular}




\section{RESULTADOS Y DISCUSIÓN}

La calidad de la predicción del volumen de exceso molar con los métodos I, II y III, utilizando las funciones de L-L, D-G, Eyk, A-B, New y Os, se calcula con la siguiente expresión [19]:

$$
\sigma_{\text {Pred }}=\left(\frac{\sum_{i}^{N_{D A T}}\left(V_{E x p, i}^{E}-V_{P r e d, i}^{E}\right)^{2}}{N_{D A T}}\right)^{1 / 2}
$$

Donde $\sigma_{P r e d}$ es la desviación de la predicción, $V_{E x p, i}^{E}$ es el volumen de exceso molar experimental, $V_{\text {Pred,i }}^{E}$ es el volumen de exceso molar calculado con los métodos I, II y III y $N_{D A T}$ es el número de datos que corresponde en la mayoría de los casos a 11 puntos en todo el rango de fracción molar. Las mezclas con menos puntos son mezclas inmiscibles que en la literatura se reportan con $N_{D A T}<11$. El mejor método para predecir $V_{M}^{E}$ en cada sistema binario, se calcula con el porcentaje de desviación promedio PD [19]:

$$
P D=\frac{100}{N_{D A T}} \sum_{i=1}^{N_{D A T}}\left|\frac{V_{E x p}^{E}-V_{P r e d}^{E}}{\left(V_{E x p}^{E}\right)_{m d}}\right|_{i}
$$

Donde $\left(V_{E x p}^{E}\right)_{m d}$ representa la mayor desviación experimental de volumen de exceso (positiva o negativa), según la tendencia observada para cada sistema binario.

Los resultados y fuentes de literatura se resumen en la Tabla 2 y se representan gráficamente en las Figuras 2-7. En la Tabla 3, se resume para los sistemas binarios el PD mínimo (mejor predicción), de cada mezcla.

Tabla 2. Desviaciones de predicción para el volumen de exceso molar a 298.15 K para las mezclas binarias con los

\begin{tabular}{|c|c|c|c|c|c|c|c|c|c|}
\hline \multirow{2}{*}{$\begin{array}{c}\text { Sistema } \\
\text { [Referencia] }\end{array}$} & \multirow[t]{2}{*}{$\sigma_{\text {Pred }}$} & \multicolumn{3}{|c|}{ Método } & \multirow{2}{*}{$\begin{array}{c}\text { Sistema } \\
\text { [Referencia] }\end{array}$} & \multirow[t]{2}{*}{$\sigma_{\text {Pred }}$} & \multicolumn{3}{|c|}{ Método } \\
\hline & & I & II & III & & & I & II & III \\
\hline \multirow{6}{*}{$\begin{array}{c}{[\mathrm{BMIM}]\left[\mathrm{PF}_{6}\right]-} \\
\text { etanol } \\
{[23]}\end{array}$} & $L-L$ & 0.113 & 0.114 & 0.084 & \multirow{6}{*}{$\begin{array}{c}{\left[\mathrm{HMIM}^{\mathrm{M}}\left[\mathrm{PF}_{6}\right] \text {-acetato }\right.} \\
\text { de metilo } \\
{[25]}\end{array}$} & $L-L$ & 0.232 & 0.251 & 0.127 \\
\hline & D-G & 0.130 & 0.132 & 0.124 & & $D-G$ & 0.347 & 0.368 & 0.293 \\
\hline & Eyk & 0.119 & 0.121 & 0.105 & & Eyk & 0.285 & 0.305 & 0.213 \\
\hline & $A-B$ & 0.148 & 0.148 & 0.148 & & $A-B$ & 0.739 & 0.737 & 0.744 \\
\hline & New & 0.158 & 0.160 & 0.184 & & New & 0.517 & 0.543 & 0.540 \\
\hline & Os & 0.133 & 0.112 & 0.122 & & Os & 0.384 & 0.313 & 0.289 \\
\hline \multirow{6}{*}{$\begin{array}{c}{[\mathrm{HMIM}]\left[\mathrm{PF}_{6}\right]-} \\
\text { etanol } \\
{[23]}\end{array}$} & $L-L$ & 0.148 & 0.149 & 0.095 & \multirow{6}{*}{$\begin{array}{c}\text { [HMIM][PF }] \text {-acetato } \\
\text { de butilo } \\
{[25]}\end{array}$} & $L-L$ & 0.123 & 0.134 & 0.082 \\
\hline & $D-G$ & 0.156 & 0.158 & 0.141 & & $D-G$ & 0.261 & 0.274 & 0.239 \\
\hline & Eyk & 0.146 & 0.148 & 0.118 & & Eyk & 0.194 & 0.205 & 0.166 \\
\hline & $A-B$ & 0.208 & 0.208 & 0.210 & & $A-B$ & 0.665 & 0.664 & 0.667 \\
\hline & New & 0.171 & 0.173 & 0.215 & & New & 0.461 & 0.476 & 0.460 \\
\hline & Os & 0.150 & 0.126 & 0.140 & & Os & 0.320 & 0.254 & 0.235 \\
\hline
\end{tabular}
métodos I, II y III 


\begin{tabular}{|c|c|c|c|c|c|c|c|c|c|}
\hline $\begin{array}{c}\text { [OMIM] }\left[\mathrm{PF}_{6}\right]- \\
\text { etanol } \\
{[23]}\end{array}$ & $\begin{array}{c}\text { L-L } \\
\text { D-G } \\
\text { Eyk } \\
\text { A-B } \\
\text { New } \\
\text { Os }\end{array}$ & $\begin{array}{l}0.075 \\
0.066 \\
0.063 \\
0.208 \\
0.062 \\
0.054\end{array}$ & $\begin{array}{l}0.076 \\
0.067 \\
0.065 \\
0.208 \\
0.064 \\
0.041\end{array}$ & $\begin{array}{l}0.029 \\
0.049 \\
0.034 \\
0.209 \\
0.105 \\
0.049\end{array}$ & $\begin{array}{c}{[\mathrm{HMIM}]\left[\mathrm{PF}_{6}\right]-} \\
\mathrm{DMC} \\
{[25]}\end{array}$ & $\begin{array}{c}\text { L-L } \\
\text { D-G } \\
\text { Eyk } \\
\text { A-B } \\
\text { New } \\
\text { Os }\end{array}$ & $\begin{array}{l}0.075 \\
0.163 \\
0.115 \\
0.670 \\
0.294 \\
0.192\end{array}$ & $\begin{array}{l}0.087 \\
0.176 \\
0.127 \\
0.669 \\
0.309 \\
0.133\end{array}$ & $\begin{array}{l}0.033 \\
0.121 \\
0.060 \\
0.674 \\
0.313 \\
0.118\end{array}$ \\
\hline $\begin{array}{c}\text { BMIM] }\left[\mathrm{PF}_{6}\right]- \\
\text { 2-butanona } \\
{[24]}\end{array}$ & $\begin{array}{c}\text { L-L } \\
D-G \\
\text { Eyk } \\
\text { A-B } \\
\text { New } \\
\text { Os }\end{array}$ & $\begin{array}{l}0.098 \\
0.233 \\
0.159 \\
0.954 \\
0.468 \\
0.298\end{array}$ & $\begin{array}{l}0.110 \\
0.256 \\
0.178 \\
0.952 \\
0.498 \\
0.226\end{array}$ & $\begin{array}{l}0.086 \\
0.203 \\
0.127 \\
0.957 \\
0.465 \\
0.194\end{array}$ & $\begin{array}{c}\mathrm{HMIM}]\left[\mathrm{PF}_{6}\right]- \\
\text { DEC } \\
{[25]}\end{array}$ & $\begin{array}{c}\text { L-L } \\
D-G \\
\text { Eyk } \\
\text { A-B } \\
\text { New } \\
\text { Os }\end{array}$ & $\begin{array}{l}0.053 \\
0.118 \\
0.055 \\
0.944 \\
0.326 \\
0.175\end{array}$ & $\begin{array}{l}0.043 \\
0.133 \\
0.066 \\
0.943 \\
0.345 \\
0.106\end{array}$ & $\begin{array}{l}0.119 \\
0.096 \\
0.061 \\
0.947 \\
0.331 \\
0.093\end{array}$ \\
\hline $\begin{array}{c}{\left[\mathrm{HMIM}^{\prime}\left[\mathrm{PF}_{6}\right]-\right.} \\
\text { 2-butanona } \\
{[25]}\end{array}$ & $\begin{array}{c}L-L \\
D-G \\
\text { Eyk } \\
\text { A-B } \\
\text { New } \\
\text { Os }\end{array}$ & $\begin{array}{l}0.116 \\
0.268 \\
0.192 \\
0.858 \\
0.490 \\
0.329 \\
\end{array}$ & $\begin{array}{l}0.136 \\
0.291 \\
0.213 \\
0.856 \\
0.517 \\
0.256 \\
\end{array}$ & $\begin{array}{l}0.045 \\
0.227 \\
0.140 \\
0.862 \\
0.492 \\
0.222 \\
\end{array}$ & $\begin{array}{c}{[\mathrm{HMIM}]\left[\mathrm{PF}_{6}\right]-} \\
\text { acetona } \\
{[25]}\end{array}$ & $\begin{array}{c}L-L \\
D-G \\
\text { Eyk } \\
A-B \\
\text { New } \\
\text { Os }\end{array}$ & $\begin{array}{l}0.192 \\
0.333 \\
0.258 \\
0.951 \\
0.543 \\
0.382 \\
\end{array}$ & $\begin{array}{l}0.222 \\
0.367 \\
0.289 \\
0.949 \\
0.583 \\
0.304 \\
\end{array}$ & $\begin{array}{l}0.059 \\
0.259 \\
0.162 \\
0.958 \\
0.555 \\
0.254 \\
\end{array}$ \\
\hline $\begin{array}{c}\text { [OMIM] }\left[\mathrm{PF}_{6}\right]- \\
\text { 2-butanona } \\
{[26]}\end{array}$ & $\begin{array}{c}\text { L-L } \\
D-G \\
\text { Eyk } \\
\text { A-B } \\
\text { New } \\
\text { Os }\end{array}$ & $\begin{array}{l}0.154 \\
0.265 \\
0.202 \\
0.965 \\
0.472 \\
0.318 \\
\end{array}$ & $\begin{array}{l}0.165 \\
0.284 \\
0.218 \\
0.963 \\
0.497 \\
0.251 \\
\end{array}$ & $\begin{array}{l}0.135 \\
0.220 \\
0.156 \\
0.968 \\
0.476 \\
0.219 \\
\end{array}$ & $\begin{array}{c}\text { [HMIM] }\left[\mathrm{PF}_{6}\right]- \\
\text { 2-pentanona } \\
{[25]}\end{array}$ & $\begin{array}{c}L-L \\
D-G \\
\text { Eyk } \\
\text { A-B } \\
\text { New } \\
\text { Os }\end{array}$ & $\begin{array}{l}0.075 \\
0.215 \\
0.143 \\
0.835 \\
0.436 \\
0.279 \\
\end{array}$ & $\begin{array}{l}0.085 \\
0.232 \\
0.158 \\
0.834 \\
0.456 \\
0.210 \\
\end{array}$ & $\begin{array}{l}0.069 \\
0.194 \\
0.121 \\
0.838 \\
0.436 \\
0.191 \\
\end{array}$ \\
\hline $\begin{array}{c}\text { [BMIM] }\left[\mathrm{PF}_{6}\right]- \\
\text { acetato de etilo } \\
{[24]}\end{array}$ & $\begin{array}{c}\text { L-L } \\
D-G \\
\text { Eyk } \\
\text { A-B } \\
\text { New } \\
\text { Os }\end{array}$ & $\begin{array}{l}0.088 \\
0.237 \\
0.160 \\
0.957 \\
0.467 \\
0.299\end{array}$ & $\begin{array}{l}0.106 \\
0.261 \\
0.181 \\
0.955 \\
0.496 \\
0.222\end{array}$ & $\begin{array}{l}0.061 \\
0.197 \\
0.114 \\
0.960 \\
0.466 \\
0.188\end{array}$ & $\begin{array}{l}{\left[\mathrm{BMIM}^{\mathrm{B}}\left[\mathrm{BF}_{4}\right]-\right.} \\
\text { nitrometano } \\
\text { [29] }\end{array}$ & $\begin{array}{c}\text { L-L } \\
\text { D-G } \\
\text { Eyk } \\
\text { A-B } \\
\text { New } \\
\text { Os }\end{array}$ & $\begin{array}{l}0.133 \\
0.189 \\
0.160 \\
0.275 \\
0.271 \\
0.210\end{array}$ & $\begin{array}{l}0.138 \\
0.194 \\
0.165 \\
0.274 \\
0.277 \\
0.176\end{array}$ & $\begin{array}{l}0.093 \\
0.172 \\
0.136 \\
0.276 \\
0.283 \\
0.172\end{array}$ \\
\hline $\begin{array}{c}{[\mathrm{HMIM}]\left[\mathrm{PF}_{6}\right]-} \\
\text { acetato de etilo } \\
{[25]}\end{array}$ & $\begin{array}{c}\text { L-L } \\
D-G \\
\text { Eyk } \\
\text { A-B } \\
\text { New } \\
\text { Os }\end{array}$ & $\begin{array}{l}0.148 \\
0.282 \\
0.212 \\
0.830 \\
0.479 \\
0.332\end{array}$ & $\begin{array}{l}0.167 \\
0.303 \\
0.231 \\
0.828 \\
0.504 \\
0.258\end{array}$ & $\begin{array}{l}0.062 \\
0.232 \\
0.149 \\
0.834 \\
0.490 \\
0.228\end{array}$ & $\begin{array}{c}\text { BMIM] }\left[\mathrm{BF}_{4}\right]- \\
\text { 1,3-dicloropropano } \\
\text { [29] }\end{array}$ & $\begin{array}{c}L-L \\
D-G \\
\text { Eyk } \\
\text { A-B } \\
\text { New } \\
\text { Os }\end{array}$ & $\begin{array}{l}0.041 \\
0.060 \\
0.045 \\
0.292 \\
0.122 \\
0.078\end{array}$ & $\begin{array}{l}0.041 \\
0.062 \\
0.046 \\
0.292 \\
0.125 \\
0.059\end{array}$ & $\begin{array}{l}0.046 \\
0.074 \\
0.054 \\
0.291 \\
0.150 \\
0.073\end{array}$ \\
\hline $\begin{array}{c}\text { [OMIM] }\left[\mathrm{PF}_{6}\right]- \\
\text { acetato de etilo } \\
{[24]}\end{array}$ & $\begin{array}{c}\text { L-L } \\
D-G \\
\text { Eyk } \\
\text { A-B } \\
\text { New } \\
\text { Os }\end{array}$ & $\begin{array}{l}0.113 \\
0.218 \\
0.161 \\
0.783 \\
0.376 \\
0.253\end{array}$ & $\begin{array}{l}0.128 \\
0.235 \\
0.176 \\
0.781 \\
0.395 \\
0.185\end{array}$ & $\begin{array}{l}0.029 \\
0.164 \\
0.088 \\
0.787 \\
0.398 \\
0.163\end{array}$ & $\begin{array}{c}\text { [BMIM] }\left[\mathrm{BF}_{4}\right]- \\
\text { etilenglicol } \\
{[29]}\end{array}$ & $\begin{array}{c}L-L \\
D-G \\
\text { Eyk } \\
A-B \\
\text { New } \\
\text { Os }\end{array}$ & $\begin{array}{l}0.054 \\
0.014 \\
0.030 \\
0.275 \\
0.065 \\
0.022\end{array}$ & $\begin{array}{l}0.056 \\
0.015 \\
0.031 \\
0.275 \\
0.063 \\
0.015\end{array}$ & $\begin{array}{l}0.054 \\
0.015 \\
0.030 \\
0.274 \\
0.064 \\
0.015\end{array}$ \\
\hline $\begin{array}{c}{\left[\mathrm{EMIM}^{2}\left[\mathrm{EtSO}_{4}\right]-\right.} \\
\text { metanol } \\
{[27]}\end{array}$ & $\begin{array}{c}\text { L-L } \\
\text { D-G } \\
\text { Eyk } \\
\text { A-B } \\
\text { New } \\
\text { Os }\end{array}$ & $\begin{array}{l}0.168 \\
0.063 \\
0.081 \\
0.438 \\
0.175 \\
0.110 \\
\end{array}$ & $\begin{array}{l}0.174 \\
0.067 \\
0.086 \\
0.437 \\
0.169 \\
0.136 \\
\end{array}$ & $\begin{array}{l}0.144 \\
0.079 \\
0.103 \\
0.444 \\
0.097 \\
0.073 \\
\end{array}$ & $\begin{array}{c}\left.\text { [BMIM][MeSO }{ }_{4}\right]- \\
\text { nitrometano } \\
{[29]}\end{array}$ & $\begin{array}{c}L-L \\
D-G \\
\text { Eyk } \\
A-B \\
\text { New } \\
\text { Os }\end{array}$ & $\begin{array}{l}0.177 \\
0.137 \\
0.142 \\
0.232 \\
0.096 \\
0.100 \\
\end{array}$ & $\begin{array}{l}0.180 \\
0.140 \\
0.145 \\
0.231 \\
0.099 \\
0.081 \\
\end{array}$ & $\begin{array}{l}0.068 \\
0.117 \\
0.093 \\
0.235 \\
0.202 \\
0.126 \\
\end{array}$ \\
\hline
\end{tabular}




\begin{tabular}{|c|c|c|c|c|c|c|c|c|c|}
\hline $\begin{array}{c}\text { EMIM] }\left[\mathrm{EtSO}_{4}\right]- \\
\text { etanol } \\
{[28]}\end{array}$ & $\begin{array}{c}\text { L-L } \\
D-G \\
\text { Eyk } \\
\text { A-B } \\
\text { New } \\
\text { Os }\end{array}$ & $\begin{array}{l}0.156 \\
0.064 \\
0.067 \\
0.290 \\
0.154 \\
0.106\end{array}$ & $\begin{array}{l}0.121 \\
0.064 \\
0.068 \\
0.290 \\
0.151 \\
0.122\end{array}$ & $\begin{array}{l}0.113 \\
0.082 \\
0.093 \\
0.294 \\
0.088 \\
0.080\end{array}$ & $\begin{array}{c}{[\mathrm{BMIM}]\left[\mathrm{MeSO}_{4}\right]-1,3-} \\
\text { dicloropropano } \\
{[29]}\end{array}$ & $\begin{array}{c}\text { L-L } \\
\text { D-G } \\
\text { Eyk } \\
\text { A-B } \\
\text { New } \\
\text { Os }\end{array}$ & $\begin{array}{l}0.118 \\
0.168 \\
0.144 \\
0.180 \\
0.238 \\
0.190\end{array}$ & $\begin{array}{l}0.120 \\
0.171 \\
0.146 \\
0.180 \\
0.241 \\
0.167\end{array}$ & $\begin{array}{l}0.092 \\
0.158 \\
0.129 \\
0.181 \\
0.250 \\
0.168\end{array}$ \\
\hline $\begin{array}{c}\text { EMIM] }\left[\mathrm{EtSO}_{4}\right]- \\
\text { 1-propanol } \\
{[27]}\end{array}$ & $\begin{array}{c}\text { L-L } \\
D-G \\
\text { Eyk } \\
\text { A-B } \\
\text { New } \\
\text { Os }\end{array}$ & $\begin{array}{l}0.086 \\
0.015 \\
0.032 \\
0.181 \\
0.090 \\
0.052\end{array}$ & $\begin{array}{l}0.087 \\
0.016 \\
0.033 \\
0.181 \\
0.089 \\
0.066 \\
\end{array}$ & $\begin{array}{l}0.052 \\
0.024 \\
0.034 \\
0.183 \\
0.042 \\
0.022\end{array}$ & $\begin{array}{c}\mathrm{MMIM}]\left[\mathrm{MeSO}_{4}\right]- \\
\text { etanol } \\
{[23]}\end{array}$ & $\begin{array}{c}\text { L-L } \\
D-G \\
\text { Eyk } \\
\text { A-B } \\
\text { New } \\
\text { Os }\end{array}$ & $\begin{array}{l}0.367 \\
0.348 \\
0.339 \\
0.459 \\
0.335 \\
0.311 \\
\end{array}$ & $\begin{array}{l}0.382 \\
0.365 \\
0.355 \\
0.458 \\
0.355 \\
0.273\end{array}$ & $\begin{array}{l}0.119 \\
0.224 \\
0.173 \\
0.468 \\
0.401 \\
0.244 \\
\end{array}$ \\
\hline $\begin{array}{c}{[\mathrm{EMIM}]\left[\mathrm{EtSO}_{4}\right]-} \\
\text { 2-propanol } \\
{[27]}\end{array}$ & $\begin{array}{c}\text { L-L } \\
D-G \\
\text { Eyk } \\
\text { A-B } \\
\text { New } \\
\text { Os }\end{array}$ & $\begin{array}{l}0.160 \\
0.077 \\
0.097 \\
0.240 \\
0.059 \\
0.035 \\
\end{array}$ & $\begin{array}{l}0.163 \\
0.079 \\
0.099 \\
0.240 \\
0.057 \\
0.041 \\
\end{array}$ & $\begin{array}{l}0.043 \\
0.058 \\
0.043 \\
0.243 \\
0.130 \\
0.065 \\
\end{array}$ & $\begin{array}{c}{[\mathrm{MMIM}]\left[\mathrm{MeSO}_{4}\right]-} \\
\text { 2-butanona } \\
{[24]}\end{array}$ & $\begin{array}{c}\text { L-L } \\
\text { D-G } \\
\text { Eyk } \\
\text { A-B } \\
\text { New } \\
\text { Os }\end{array}$ & $\begin{array}{l}0.167 \\
0.219 \\
0.191 \\
0.386 \\
0.291 \\
0.232 \\
\end{array}$ & $\begin{array}{l}0.177 \\
0.228 \\
0.199 \\
0.385 \\
0.303 \\
0.201 \\
\end{array}$ & $\begin{array}{l}0.084 \\
0.182 \\
0.136 \\
0.389 \\
0.335 \\
0.200 \\
\end{array}$ \\
\hline $\begin{array}{c}{\left[{\mathrm{BMIM}]\left[\mathrm{BF}_{4}\right]-}^{\text {etanol }}\right.} \\
\text { [29] }\end{array}$ & $\begin{array}{c}\text { L-L } \\
D-G \\
\text { Eyk } \\
\text { A-B } \\
\text { New } \\
\text { Os }\end{array}$ & $\begin{array}{l}0.092 \\
0.099 \\
0.090 \\
0.272 \\
0.113 \\
0.093\end{array}$ & $\begin{array}{l}0.094 \\
0.101 \\
0.092 \\
0.272 \\
0.116 \\
0.069\end{array}$ & $\begin{array}{l}0.050 \\
0.084 \\
0.064 \\
0.275 \\
0.160 \\
0.084\end{array}$ & $\begin{array}{l}\left.\text { 2-pIM][MeSO }{ }_{4}\right]- \\
\text { [24] }\end{array}$ & $\begin{array}{c}\text { L-L } \\
\text { D-G } \\
\text { Eyk } \\
\text { A-B } \\
\text { New } \\
\text { Os }\end{array}$ & $\begin{array}{l}0.210 \\
0.168 \\
0.170 \\
0.398 \\
0.126 \\
0.122\end{array}$ & $\begin{array}{l}0.218 \\
0.176 \\
0.177 \\
0.398 \\
0.134 \\
0.088\end{array}$ & $\begin{array}{l}0.044 \\
0.107 \\
0.067 \\
0.405 \\
0.245 \\
0.123\end{array}$ \\
\hline $\begin{array}{c}\text { [BMIM] }\left[\mathrm{MeSO}_{4}\right]- \\
\text { etanol } \\
{[23]}\end{array}$ & $\begin{array}{c}\text { L-L } \\
\text { D-G } \\
\text { Eyk } \\
\text { A-B } \\
\text { New } \\
\text { Os }\end{array}$ & $\begin{array}{l}0.209 \\
0.118 \\
0.138 \\
0.308 \\
0.060 \\
0.058\end{array}$ & $\begin{array}{l}0.212 \\
0.122 \\
0.142 \\
0.308 \\
0.058 \\
0.050\end{array}$ & $\begin{array}{l}0.060 \\
0.087 \\
0.066 \\
0.313 \\
0.180 \\
0.096\end{array}$ & $\begin{array}{c}{[\mathrm{MMIM}]\left[\mathrm{MeSO}_{4}\right]-} \\
\text { acetato de etilo } \\
{[24]}\end{array}$ & $\begin{array}{c}\text { L-L } \\
\text { D-G } \\
\text { Eyk } \\
\text { A-B } \\
\text { New } \\
\text { Os }\end{array}$ & $\begin{array}{l}0.139 \\
0.162 \\
0.145 \\
0.258 \\
0.198 \\
0.162\end{array}$ & $\begin{array}{l}0.143 \\
0.166 \\
0.149 \\
0.257 \\
0.203 \\
0.135\end{array}$ & $\begin{array}{l}0.065 \\
0.154 \\
0.116 \\
0.259 \\
0.272 \\
0.168\end{array}$ \\
\hline
\end{tabular}

Tabla 3. Valor PD mínimo para cada mezcla binaria

\begin{tabular}{|c|c|c|c|}
\hline \multirow[t]{2}{*}{ Mezcla } & \multicolumn{3}{|c|}{ PD mínimo ${ }^{a}$} \\
\hline & Valor & Método & Función \\
\hline$[\mathrm{BMIM}]\left[\mathrm{PF}_{6}\right]$-etanol & 12.9 & III & L-L \\
\hline$[\mathrm{HMIM}]\left[\mathrm{PF}_{6}\right]$-etanol & 11.8 & III & $L-L$ \\
\hline [OMIM] $\left[\mathrm{PF}_{6}\right]$-etanol & 4.5 & III & L-L \\
\hline$\left[\mathrm{BMIM}_{[}\left[\mathrm{PF}_{6}\right]-2\right.$-butanona & 3.1 & III & L-L \\
\hline$[\mathrm{HMIM}]\left[\mathrm{PF}_{6}\right]$-2-butanona & 1.7 & III & L-L \\
\hline [OMIM] $\left[\mathrm{PF}_{6}\right]$-2-butanona & 4.3 & I & L-L \\
\hline$[\mathrm{BMIM}]\left[\mathrm{PF}_{6}\right]$-acetato de etilo & 2.2 & III & L-L \\
\hline [HMIM] $\left[\mathrm{PF}_{6}\right]$ - acetato de etilo & 2.5 & III & $L-L$ \\
\hline [OMIM][PF $\left.{ }_{6}\right]$ - acetato de etilo & 1.2 & III & L-L \\
\hline$[\mathrm{EMIM}]\left[\mathrm{EtSO}_{4}\right]$-metanol & 4.6 & III & Os \\
\hline$[\mathrm{EMIM}]\left[\mathrm{EtSO}_{4}\right]$-etanol & 6.9 & II & $D-G$ \\
\hline [EMIM][EtSO $\left.{ }_{4}\right]$-1-propanol & 2.8 & 1 & D-G \\
\hline [EMIM] $\left[\mathrm{EtSO}_{4}\right]$-2-propanol & 4.5 & I & Os \\
\hline$[\mathrm{BMIM}]\left[\mathrm{BF}_{4}\right]$-etanol & 6.8 & III & L-L \\
\hline [BMIM] [MeSO 4 ]-etanol & 5.8 & II & Os \\
\hline
\end{tabular}




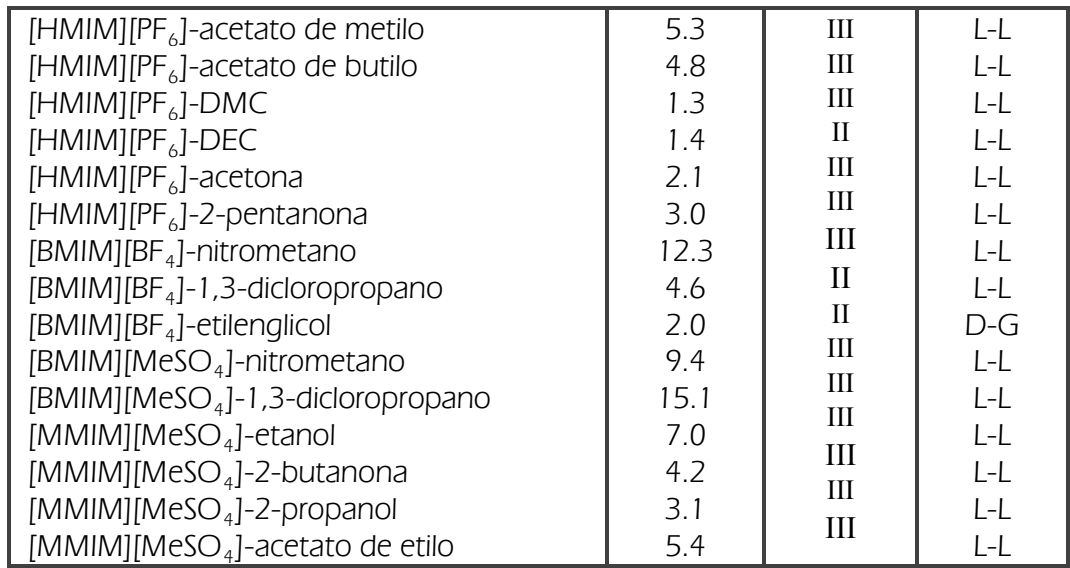

${ }^{a}$ Corresponde al menor valor con la ecuación (8) para cada mezcla binaria

Las incertidumbres reportadas en literatura para datos experimentales de índice de refracción y volumen de exceso molar de las mezclas binarias consideradas en este estudio, son mejores que 0.0001 y $0.001 \mathrm{~cm}^{3} \mathrm{~mol}^{-1}$ respectivamente.

\subsection{GRUPO 1. EFECTO DE LONGITUD DE CADENA DEL CATIÓN DEL LíOUIDO IÓNICO}
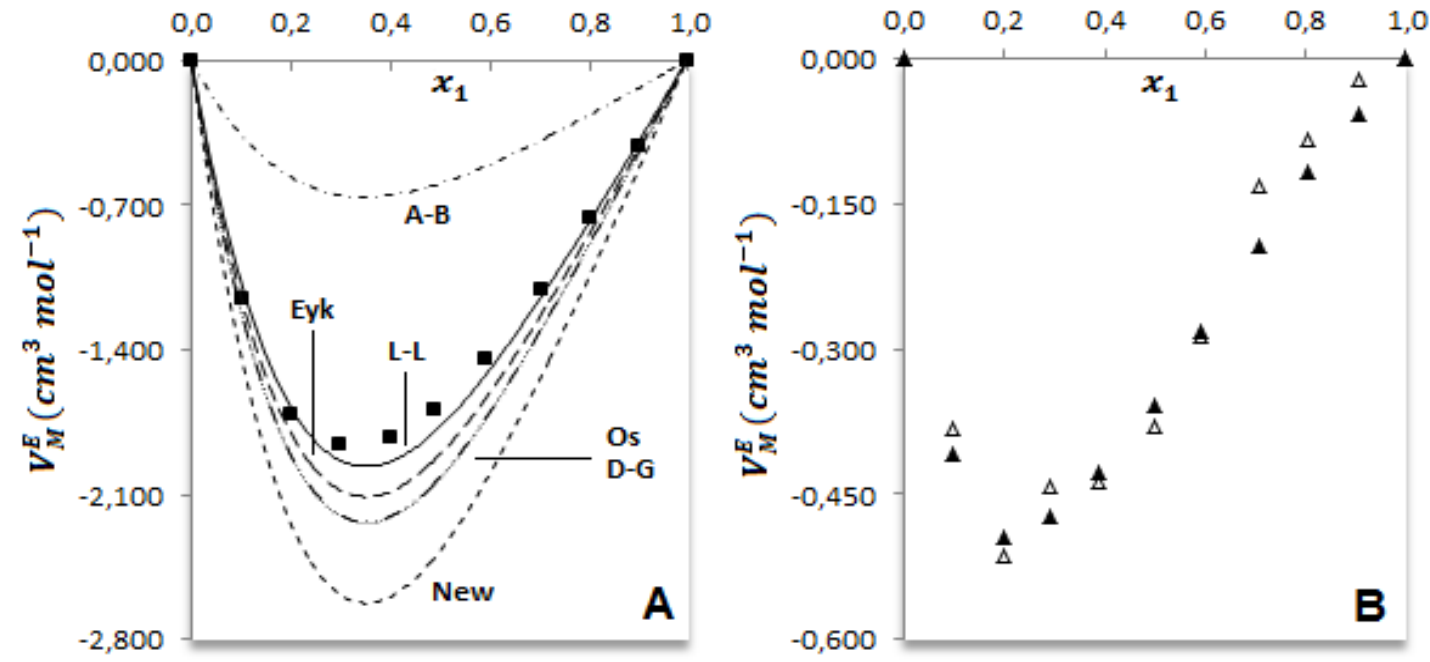

Figura 2. (A) Sistema binario $[H M I M]\left[\mathrm{PF}_{6}\right](1)+$ acetato de etilo(2), a: datos experimentales [25], líneas de predicción del $V_{M}^{E}$ funciones L-L, D-G, Eyk, A-B, New y Os, método III. (B) Sistema binario [OMIM][PF $](1)+$ etanol(2), $\mathbf{\Delta}$ : datos experimentales [23], $\triangle$ : predicción función L-L método III.

En este grupo, se analizan nueve mezclas binarias de tres líquidos iónicos (BMIM-PF ${ }_{6}$, HMIM$\mathrm{PF}_{6}$ y OMIM-PF 6 ), donde se deja fija la estructura del anión (hexafluorofosfato), y se varía la 
longitud de la cadena alquílica del catión de imidazolio (butil, hexil y octil), mezclados cada uno con etanol, 2-butanona y acetato de etilo. A $298.15 \mathrm{~K}$, se reportan volúmenes de exceso molar negativos para las nueve mezclas [23], [24], [25] y [26].

Las menores desviaciones de predicción de volumen de exceso molar, se obtienen con la función L-L. La función A-B $\left(f\left(n_{D}\right)=n_{D}\right)$, no predice adecuadamente el volumen de exceso molar, es una función que considera la mezcla como una mezcla ideal y en este grupo, las desviaciones de la idealidad en volumen de exceso molar reportadas son hasta de -2.099 $\mathrm{cm}^{3} \mathrm{~mol}^{-1}$ en la mezcla [BMIM] [PF ${ }_{6}$-acetato de etilo [24]. En la Figura 2 A, se compara para la mezcla binaria [HMIM] $\left[\mathrm{PF}_{6}\right]$-acetato de etilo, todas las funciones, usando el método III para la predicción del $V_{M}^{E}$. Algunos autores consideran la función New como representativas de la idealidad del índice de refracción en mezclas binarias [30].

El volumen de exceso molar negativo en las mezclas binarias BMIM-PF, HMIM-PF $_{6}$ y OMIM-PF con etanol, indica que las interacciones entre las moléculas de líquido iónico y etanol son más fuertes que las interacciones entre moléculas de los componentes puros; a $298.15 \mathrm{~K}$ se reporta un rango de inmiscibilidad en fracción molar de líquido iónico desde 0.0056 hasta 0.4960 para la mezcla [BMIM][PF $]$-etanol [23]. Las otras dos mezclas son miscibles en todo el rango de fracción molar, y el aumento de longitud de cadena alquílica del catión del líquido iónico de imidazolio desde butil hasta octil afecta la polaridad del líquido iónico favoreciendo su miscibilidad con etanol. En la Figura 2B, se comparan las predicciones con la función L-L método III para la mezcla binaria [OMIM][PF$\left.{ }_{6}\right]$-etanol. Para esta mezcla en particular, la magnitud del volumen de exceso molar es pequeño en comparación con el volumen molar de la mezcla, $V_{M}$, que se calcula con la siguiente expresión:

$$
V_{M}=\frac{x_{1} M_{1}+x_{2} M_{2}}{\rho}
$$

La magnitud de $V_{M}^{E}$ oscila entre 0.01 y $0.51 \%$ de los valores de $V_{M}$ en todo el rango de fracción molar. Una representación de $V_{M}$ en función de $x_{1}$ para la mezcla binaria [OMIM][PF ${ }_{6}$ ]-etanol, muestra una relación lineal entre el volumen molar y la fracción molar de líquido iónico a 298.15 $\mathrm{K}$ con un coeficiente de correlación de 0.99999 .

En la Figura 3 A, se muestra para la mezcla $[\mathrm{BMIM}]\left[\mathrm{PF}_{6}\right]-2-$-butanona, la predicción del $V_{M}^{E}$ con la función L-L métodos I, II y III. El valor PD mínimo calculado con la ecuación (8) es: método I= 3.6, método II=3.9 y método III=3.1. El método isorefractivo se favorece en este caso, por la proximidad del índice de refracción de los componentes puros a $298.15 \mathrm{~K}$, reportados en la literatura: 1.37618 y 1.40937 para el líquido iónico y la cetona, respectivamente [24].

Las mezclas binarias líquido iónico con 2-butanona a $298.15 \mathrm{~K}$ son miscibles en todo el rango de fracción molar y sus desviaciones negativas del volumen de exceso molar se afectan con el aumento de longitud de cadena alquílica del catión del líquido iónico. A composición equimolar $x_{1}=0.5$ el volumen de exceso molar reportado es $-1.877 \mathrm{~cm}^{3} \mathrm{~mol}^{-1},-1.664 \mathrm{~cm}^{3} \mathrm{~mol}^{-1} \mathrm{y}-$ 
$1.890 \mathrm{~cm}^{3} \mathrm{~mol}^{-1}$ para las mezclas binarias [BMIM] $\left[\mathrm{PF}_{6}\right]$-2-butanona, [HMIM] [PF 6 ]-2-butanona y [OMIM] $\left[\mathrm{PF}_{6}\right]$-2-butanona respectivamente [24], [25] y [26].

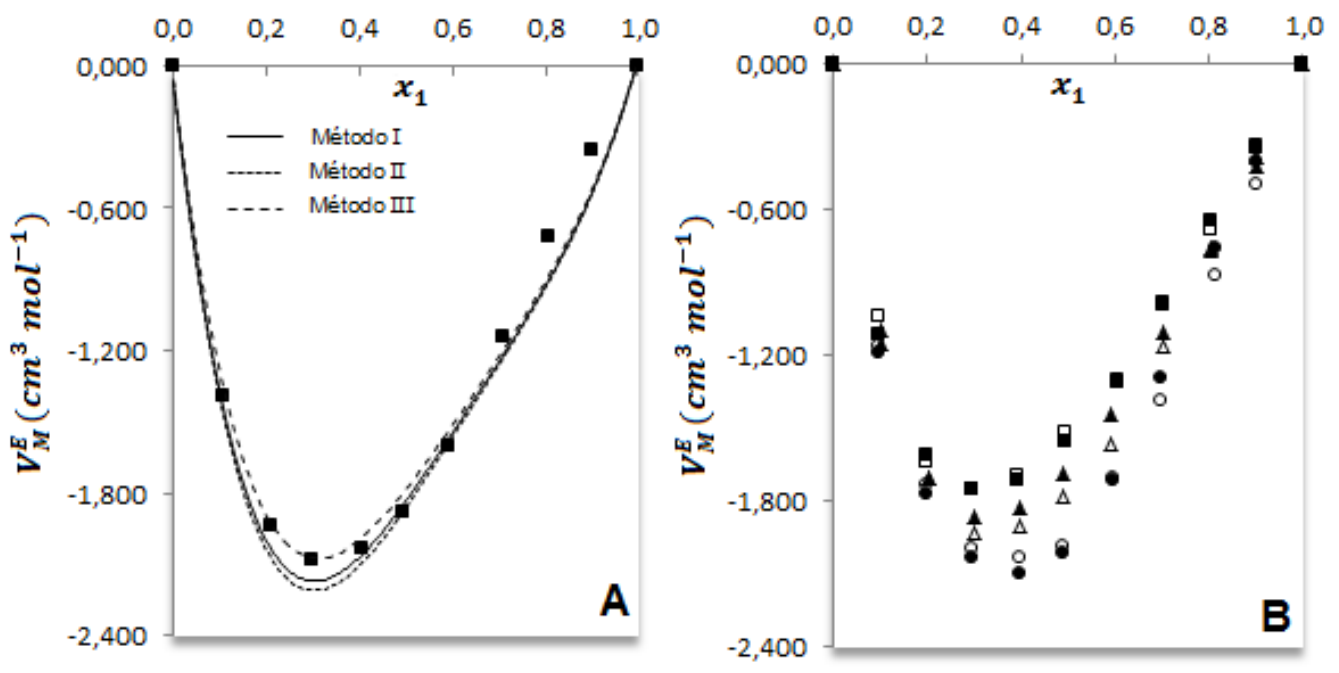

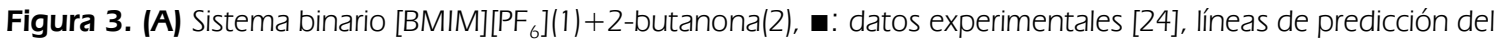
$V_{M}^{E}$ función L-L, método I, II y III. (B) Sistemas binarios líquido iónico con acetato de etilo, predicción con función LL, método III; [BMIM] [PF $]$ ] (1) + acetato de etilo(2): •: datos experimentales [24], o:datos de predicción; $[\mathrm{HMIM}]\left[\mathrm{PF}_{6}\right](1)+$ acetato de etilo(2): $\mathbf{\Delta}$ : datos experimentales [25], $\triangle$ :datos de predicción y [OMIM] [PF 6 ] (1)+ acetato de etilo(2): $\mathbf{~}:$ datos experimentales [24], $\square:$ datos de predicción.

Las mezclas binarias líquido iónico con acetato de etilo a $298.15 \mathrm{~K}$, son miscibles en todo el rango de fracción molar y sus desviaciones del volumen de exceso molar se hacen más negativas con la disminución de longitud de cadena alquílica del catión del líquido iónico [24] y [25]. El empaquetamiento del acetato de etilo en el líquido iónico durante el proceso de mezcla, se ve favorecido por la disminución de la cadena alquílica que permite una mayor interacción entre los electrones libres de los oxígenos del éster y el catión de imidazolio.

En la Figura 3B, se compara el volumen de exceso molar de las mezclas líquido iónico con acetato de etilo y su predicción con la función L-L método III.

\subsection{GRUPO 2. EFECTO DE LONGITUD DE CADENA DEL ALCOHOL}

En este grupo, se analizan cuatro mezclas binarias del líquido iónico EMIM-EtSO ${ }_{4}$ con los alcoholes metanol, etanol, 1-propanol y 2-propanol. A $298.15 \mathrm{~K}$, se reportan volúmenes de exceso molar negativos en todo el rango de fracción molar para las cuatro mezclas [27] y [28]. El volumen de exceso molar se hace menos negativo con el aumento de longitud de cadena del alcohol desde metanol hasta 1-propanol. Para el sistema binario [EMIM][EtSO ${ }_{4}$-metanol, a una fracción molar de líquido iónico de aproximadamente 0.2 se reporta un mínimo de $V_{M}^{E}=-0.912$ $\mathrm{cm}^{3} \mathrm{~mol}^{-1}$ [27]. Los valores mínimos reportados para los sistemas binarios [EMIM][EtSO ${ }_{4}$ ]-etanol 
y $[\mathrm{EMIM}]\left[\mathrm{EtSO}_{4}\right]-1$-propanol son $-0.672 \quad \mathrm{~cm}^{3} \mathrm{~mol}^{-1}$ [28] y $\quad-0.406 \quad \mathrm{~cm}^{3} \mathrm{~mol}^{-1}$ [27], respectivamente. Estos valores negativos se explican en términos de interacciones moleculares más eficientes en las mezclas que en los líquidos puros, como consecuencia de las interacciones dipolo-dipolo entre el alcohol y el líquido iónico [27].

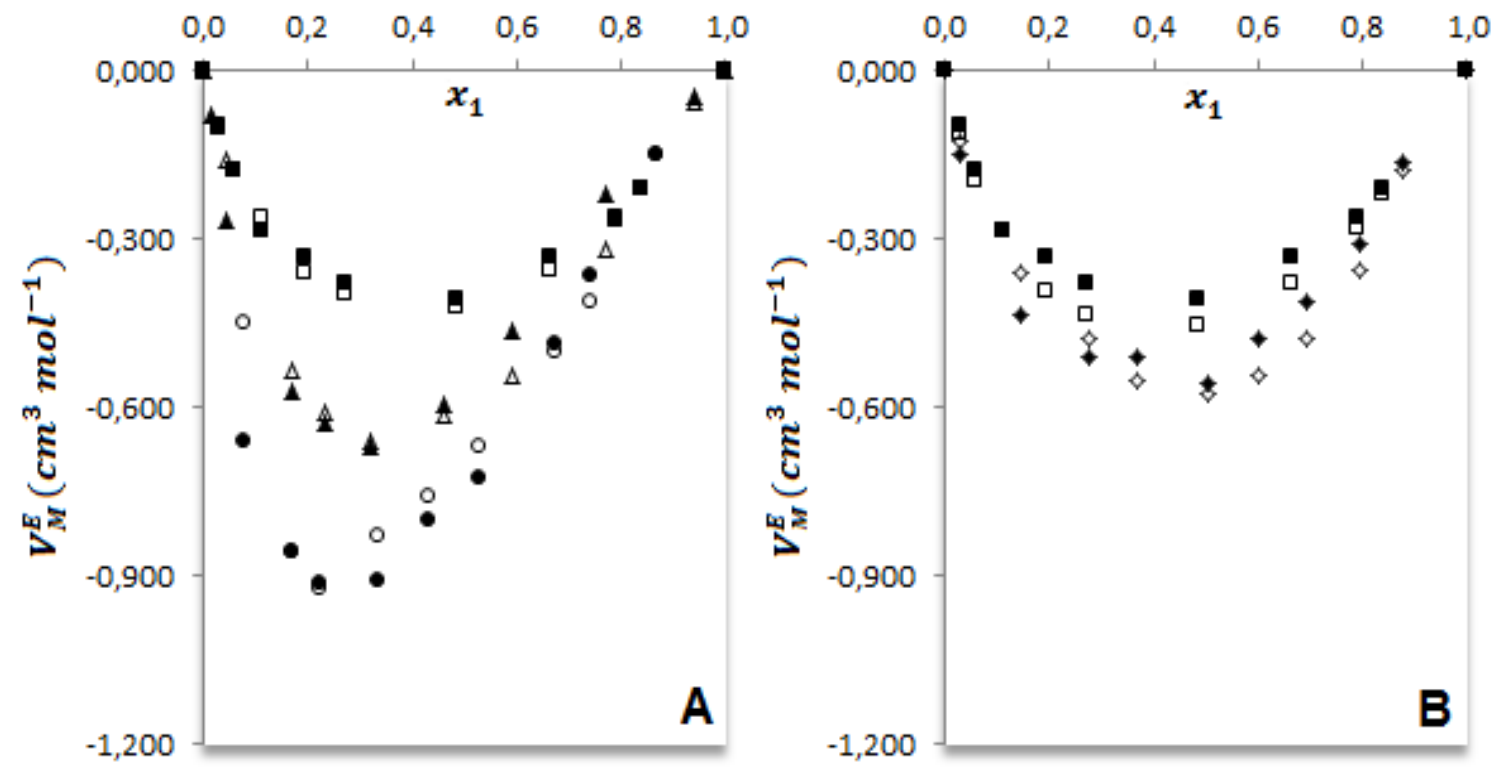

Figura 4. (A) Sistemas binarios EMIM-EtSO ${ }_{4}$ con alcohol; $[\mathrm{EMIM}]\left[\mathrm{EtSO}_{4}\right](1)+$ metanol(2): $\bullet$ : datos experimentales [27], o:datos de predicción función Os método III; [EMIM] [EtSO $]$ ](1)+etanol(2): $\mathbf{\Lambda}$ : datos experimentales [28], $\triangle$ :datos de predicción función D-G método II y [EMIM] [EtSO $]$ (1)+1-propanol(2): $\mathbf{a}$ : datos experimentales [27], $\square$ : datos de predicción función D-G método I. (B) Sistemas binarios EMIM-EtSO ${ }_{4}$ con alcohol; [EMIM] [EtSO $\left.{ }_{4}\right](1)+1$ propanol(2): a: datos experimentales [27], ם: datos de predicción función Eyk método IIy [EMIM] [EtSO $\left.{ }_{4}\right](1)+2-$ propanol(2): $\bullet$ : datos experimentales [27], $\downarrow$ :datos de predicción función Eyk método III.

Con el aumento de longitud de cadena del alcohol, el valor más negativo de volumen de exceso molar se reporta a una fracción molar más rica en líquido iónico de imidazolio. Estos efectos se muestran en la Figura 4A, y las mejores predicciones para estas mezclas (Tabla 3), se reportan con las funciones Os y D-G.

En la Figura 4B, se muestran las predicciones de volumen de exceso molar con la función Eyk método II para la mezcla[EMIM][EtSO $\left.{ }_{4}\right]-1$-propanol y función Eyk método III para la mezcla [EMIM] [EtSO $]$ ]-2-propanol. El volumen de exceso molar es más negativo para el alcohol secundario que para el alcohol primario. Al comparar las Figuras 4A y 4B para el binario

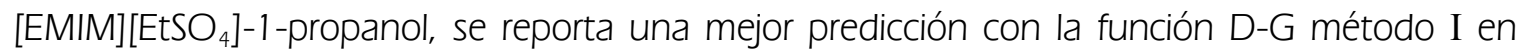
comparación con la función Eyk método II. En la Tabla 2, se muestran valores de $\sigma_{\text {Pred }}$ (ecuación 7), de 0.015 y 0.033 para D-G método I y Eyk método II, respectivamente. 


\subsection{GRUPO 3. EFECTO DEL TIPO DE ANIÓN DEL LÍOUIDO IÓNICO}

El efecto del tipo de anión del líquido iónico se reporta en tres mezclas binarias: [BMIM] $\left[\mathrm{BF}_{4}\right]$ etanol, [BMIM] [PF 6 -etanol y [BMIM] $\left[\mathrm{MeSO}_{4}\right]$-etanol. Se mantiene la estructura del catión de imidazolio y se varía la estructura del anión; a 298.15 K se reportan volúmenes de exceso molar negativos, los binarios con metilsulfato y tetrafluoroborato son miscibles en todo el rango de fracción molar, y la mezcla de BMIM-PF 6 con etanol presenta una zona de inmiscibilidad (sección 2.1).

El volumen de exceso molar se afecta por el tipo de anión del líquido iónico. La mayor desviación se reporta para el binario con el anión metilsulfato que con su grupo metilo, favorece la interacción entre el líquido iónico y la zona apolar del etanol. La menor desviación se reporta para el binario con hexafluorofosfato que a $298.15 \mathrm{~K}$ es inmiscible a fracción molar de líquido iónico desde 0.0056 hasta 0.4960 [23] y [29]. En la Figura 5 A, se muestra la mejor predicción (Tabla 3), para las mezclas binarias [BMIM] [BF $\left.{ }_{4}\right]$-etanol, [BMIM] [PF $]$-etanol y [BMIM][MeSO $\left.{ }_{4}\right]-$ etanol.

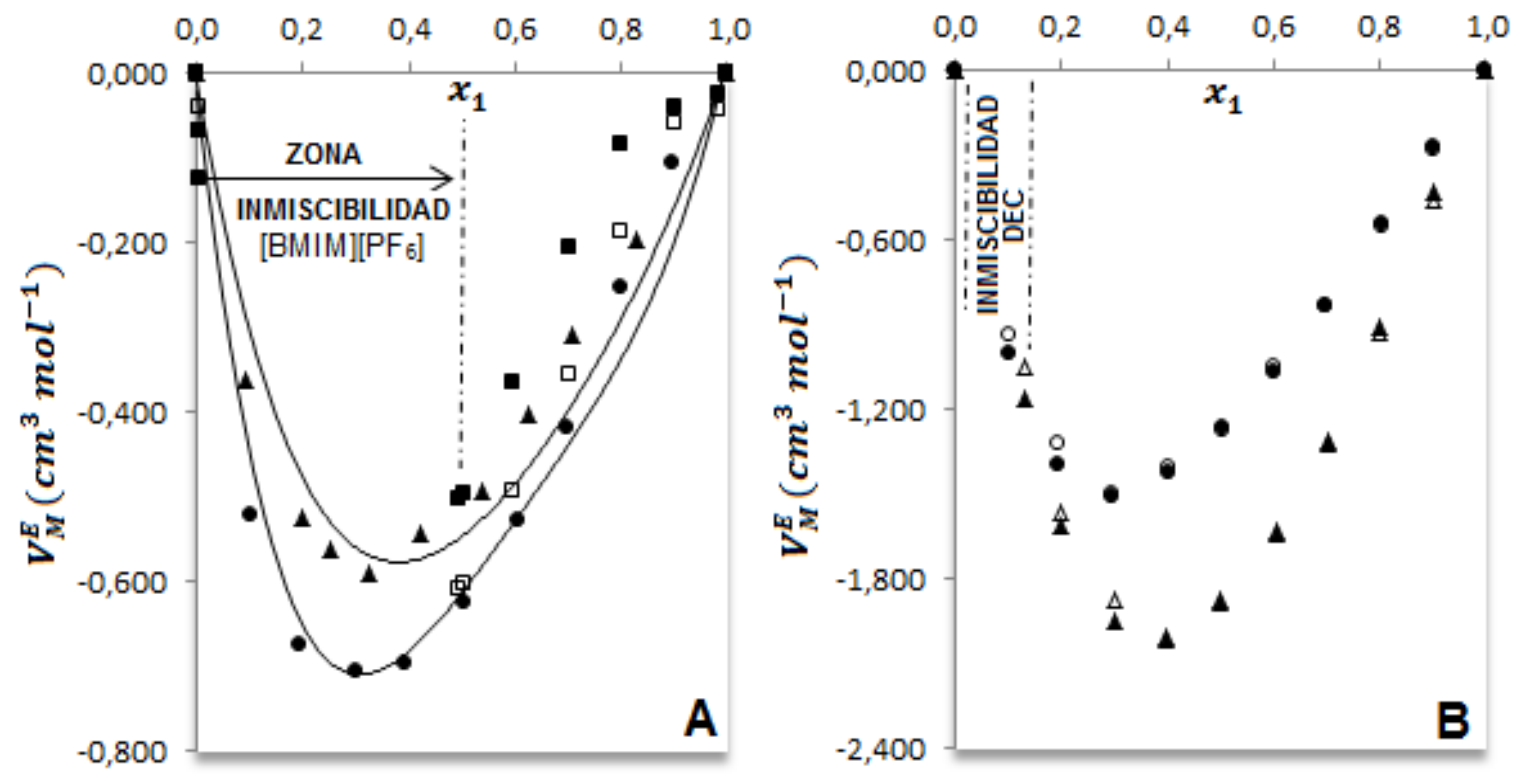

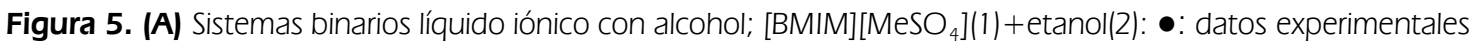
[23], __ línea de predicción función Os método II; [BMIM] [BF 4 ](1)+etanol(2): $\mathbf{\Delta}$ : datos experimentales [29], línea de predicción función L-L método IIIy [BMIM][PF $\left.{ }_{6}\right](1)+$ etano(2): $\mathbf{a}$ : datos experimentales [23], $\square$ : datos de predicción función L-L método III. (B) Sistemas binarios $\mathrm{HMIM}-\mathrm{PF}_{6}$ con ésteres de carbonato; $\left[\mathrm{HMIM}_{[}\left[\mathrm{PF}_{6}\right](1)+\mathrm{DMC}(2): \bullet:\right.$ datos experimentales [25], o:datos de predicción función L-L método III; $[\mathrm{HMIM}]\left[\mathrm{PF}_{6}\right](1)+\mathrm{DEC}(2): \mathbf{\Delta}:$ datos experimentales [25], $\triangle$ :datos de predicción función L-L método II. 


\subsection{GRUPO 4. EFECTO DE LA NATURALEZA DEL ÉSTER}

En este grupo, se analizan cinco mezclas binarias del líquido iónico HMIM-PF 6 con los ésteres: acetato de metilo, acetato de etilo, acetato de butilo y con dos ésteres de carbonato (DMC y DEC). A $298.15 \mathrm{~K}$, se reportan volúmenes de exceso molar negativos, las interacciones moleculares entre el líquido iónico con los ésteres de carbonato y acetatos son mayores que las interacciones moleculares de los componentes puros [25]. Todas las mezclas son miscibles en el rango de fracción molar, con excepción de la mezcla con carbonato de dietilo que es inmiscible a fracciones de líquido iónico entre 0.0098 y 0.1298 [25].

Las menores desviaciones de predicción de volumen de exceso molar, se obtienen con la función L-L; para los ésteres de carbonato, el $V_{M}^{E}$ se hace más negativo con el aumento de la longitud de cadena del éster. En el caso de los acetatos, el $V_{M}^{E}$ se hace más negativo desde el metilo al etilo, y menos negativo desde el etilo al butilo, lo cual indica que el empaquetamiento del acetato de etilo en la estructura del líquido iónico, es más efectivo comparado con el de los acetatos de metilo y butilo. En la Figura 5B, se comparan los volúmenes de exceso molar para las mezclas binarias de HMIM-PF 6 con los ésteres de carbonato y su predicción función L-L método III para DMC y método II para DEC, con desviaciones de predicción 1.3 y 1.4, respectivamente.

\subsection{GRUPO 5. EFECTO DE LONGITUD DE CADENA DE LA CETONA}

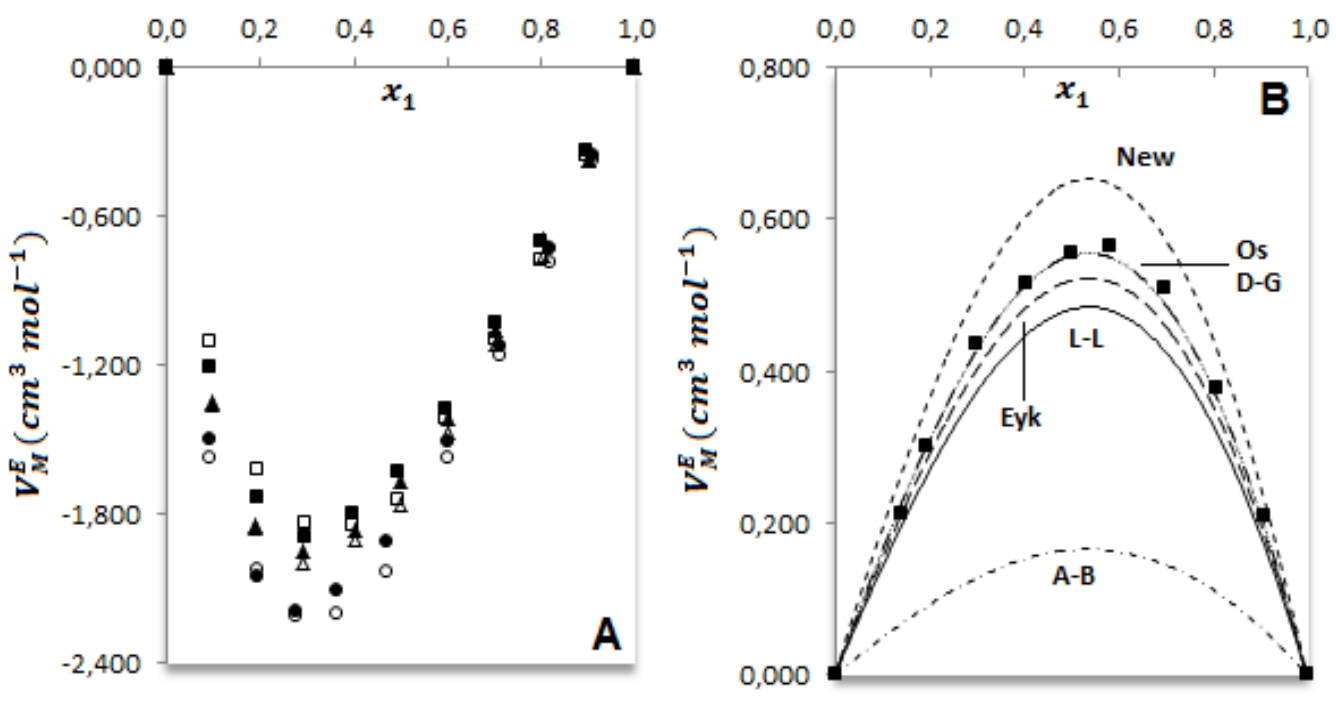

Figura 6. (A) Sistemas binarios HMIM-PF 6 con cetonas; $[H M I M]\left[P F_{6}\right](1)+$ acetona(2): $\bullet$ : datos experimentales [25], ○:datos de predicción función L-L método III; [HMIM] [PF 6 ](1)+2-butanona(2):

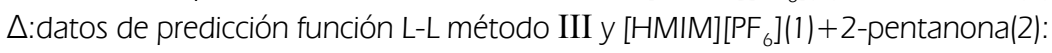

$\boldsymbol{\Delta}$ : datos experimentales [25],

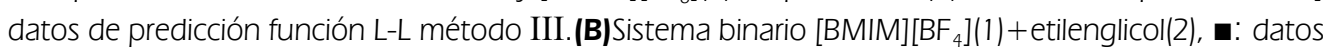
experimentales [29], líneas de predicción del $V_{M}^{E}$ funciones L-L, D-G, Eyk, A-B, New y Os, método II. 
Se reportan tres mezclas binarias del líquido iónico HMIM-PF 6 con acetona, 2-butanona y 2pentanona con volumen de exceso molar negativo en todo el rango de fracción molar a 298.15 K [25]. La contracción del $V_{M}^{E}$ se hace menor con el aumento de la longitud de cadena de la cetona desde 3 hasta 5 carbonos. El aumento de la cadena alquílica a lado y lado del enlace $(\mathrm{C}=\mathrm{O})$, disminuye la efectividad del empaquetamiento de la cetona en el líquido iónico y por consiguiente, una disminución de la desviación del volumen de exceso. En la Figura 6 A, se compara el volumen de exceso molar de las mezclas binarias [HMIM] [PF 6 -cetona, [HMIM] [PF $\left.{ }_{6}\right]$ 2-butanona y [HMIM] [PF 6 -2-pentanona y su predicción con la función L-L método III.

\subsection{GRUPO 6. EFECTO DE LA NATURALEZA DEL SOLVENTE MOLECULAR}

Este grupo se divide en tres subgrupos: el primero con el líquido iónico BMIM-BF ${ }_{4}$ mezclado con cuatro solventes diferentes: etanol, nitrometano, 1,3-dicloropropano y etilenglicol. A 298.15 K, se reportan volúmenes de exceso molar negativos para las mezclas [BMIM] $\left[\mathrm{BF}_{4}\right]$-etanol, $[\mathrm{BMIM}]\left[\mathrm{BF}_{4}\right]$-nitrometano, $[\mathrm{BMIM}]\left[\mathrm{BF}_{4}\right]$-1,3-dicloropropano y volúmenes de exceso molar positivos para la mezcla [BMIM] $\left[\mathrm{BF}_{4}\right.$ ] etilenglicol [29]. La función $L t$ predice adecuadamente $V_{M}^{E}$ negativos; con las funciones Os y D-G se obtiene la mejor predicción para el $V_{M}^{E}$ positivo de la mezcla binaria con etilenglicol, aunque con las otras funciones L-L, Eyk, A-B y New también se predice adecuadamente el signo de $V_{M}^{E}$. En la Figura 6B, para la mezcla binaria [BMIM] $\left[\mathrm{BF}_{4}\right]$ etilenglicol, se comparan todas las funciones, usando el método II para la predicción del $V_{M}^{E}$. Se observa que la función no influye significativamente en el signo y forma de la curva de predicción, pero sí afecta su magnitud.

Al comparar el $V_{M}^{E}$ negativo de las mezclas [BMIM][BF $\left.{ }_{4}\right]$-etanol, [BMIM] $\left[\mathrm{BF}_{4}\right]$-nitrometano, [BMIM] $\left[\mathrm{BF}_{4}\right]$-1,3-dicloropropano, los valores más negativos se reportan para la mezcla con el solvente nitrogenado [29]. Estos tres solventes presentan un factor de empaquetamiento adecuado en el líquido iónico, causando una contracción del volumen. En la mezcla [BMIM] $\left[\mathrm{BF}_{4}\right]$-etilenglicol, el empaquetamiento no es adecuado y el volumen final de la mezcla a $298.15 \mathrm{~K}$, es superior a la suma del volumen de los dos líquidos puros.

El seguncia del radical alquilo en el anión del líquido iónico causa $V_{M}^{E}$ más negativos en mezclas

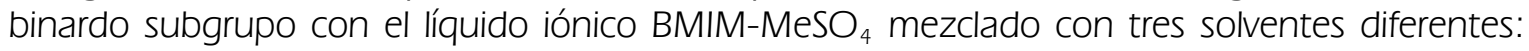
etanol, nitrometano y 1,3-dicloropropano. A $298.15 \mathrm{~K}$ en todo el rango de fracción molar, las tres mezclas presentan volúmenes de exceso molar negativos [23] y [29]. Al comparar el $V_{M}^{E}$, los valores más negativos se reportan para la mezcla [BMIM] $\left[\mathrm{MeSO}_{4}\right]$-etanol, incluso cuando se compara con los valores reportados para la mezcla [BMIM] $\left[\mathrm{BF}_{4}\right]$-etanol. Esto indica que la presenias de líquido iónico de imidazolio con etanol. En la Figura 7 A, se muestra la mejor predicción (Tabla 3), para las mezclas binarias [BMIM][MeSO$\left.{ }_{4}\right]$-etanol, [BMIM][MeSO$\left.{ }_{4}\right]-$ nitrometano y [BMIM][MeSO 4 ]-1,3-dicloropropano.

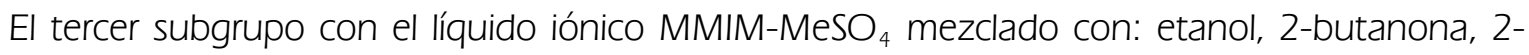
propanol y acetato de etilo. A $298.15 \mathrm{~K}$, se reportan volúmenes de exceso molar negativos, las mezclas con alcohol son miscibles, mientras que la mezcla con 2-butanona es inmiscible a fracciones molares de líquido iónico entre 0.0041 y 0.7036 y la mezcla con acetato de etilo inmiscible entre 0.0000 y 0.8397 [24]. 


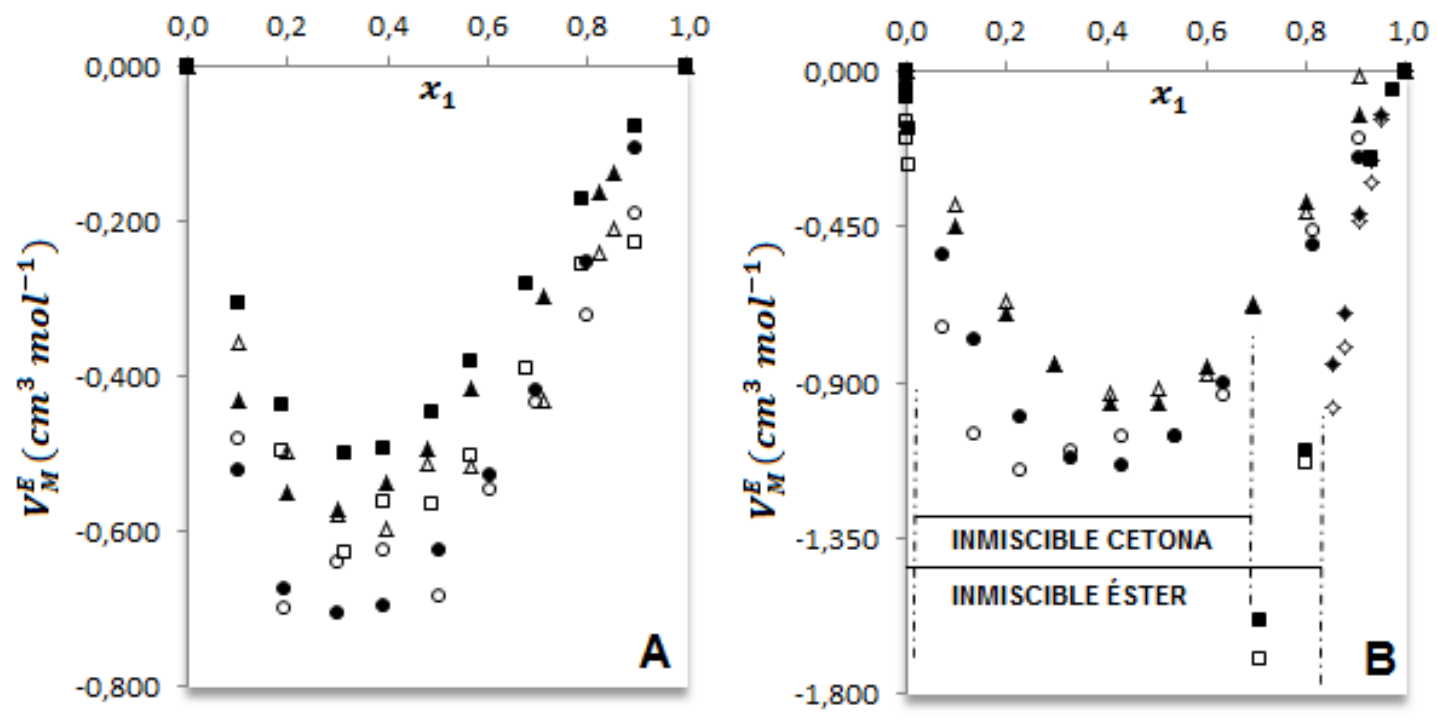

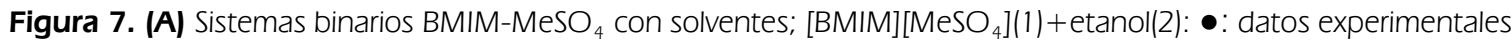
[23], o:datos de predicción función Os método II; [BMIM] [MeSO 4 ](1)+nitrometano(2): $\mathbf{\Delta}$ : datos experimentales

[29], $\Delta$ :datos de predicción función L-L método III y [BMIM][MeSO $\left.{ }_{4}\right](1)+1$,3-dicloropropano(2):-a: datos

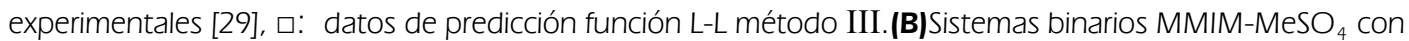
solventes; [MMIM][MeSO $\left.{ }_{4}\right](1)+$ etanol(2): •: datos experimentales [23], ○:datos de predicción función L-L método III; [MMIM] [MeSO 4 ](1)+2-propanol(2): $\mathbf{\Lambda}$ : datos experimentales [24], $\Delta$ :datos de predicción función L-L método III; [MMIM][MeSO $\left.{ }_{4}\right]$ (1)+2-butanona(2): $\mathbf{m}$ : datos experimentales [24], $\square$ : datos de predicción función L-L método

III y [MMIM] $\left[\mathrm{MeSO}_{4}\right](1)+$ acetato de etilo(2): $\bullet$ : datos experimentales [24], $\diamond$ :datos de predicción función L-L método III.

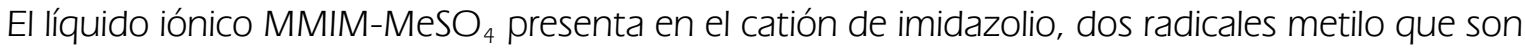
pequeños en comparación con los radicales de otros líquidos iónicos considerados en este estudio. Los radicales alquílicos pequeños en el catión de imidazolio, disminuye el tamaño de la

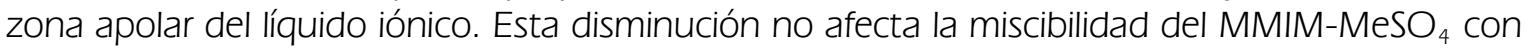
etanol y 2-propanol a 298.15 K; incluso se observa la misma tendencia reportada en la sección 2.2: el volumen de exceso molar se hace menos negativo con el aumento de longitud de cadena del alcohol. En contraste, la disminución de la longitud de cadena de los radicales del líquido iónico sí afecta la solubilidad de la 2-butanona y del acetato de etilo en $\mathrm{MMIM}^{\mathrm{M}} \mathrm{MeSO}_{4}$ [24]. En la Figura 7B, se compara el $V_{M}^{E}$ para las mezclas binarias [MMIM][MeSO${ }_{4}$ ]-etanol,

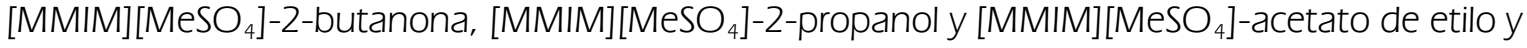
su predicción con la función L-L método III.

\section{CONCLUSIONES}

Se expuso la relación que existe entre el volumen de exceso molar y el índice de refracción de mezclas binarias. Las mejores predicciones del volumen de exceso molar para las 30 mezclas 
binarias líquido iónico de imidazolio con solvente molecular desde índices de refracción, se reportan con la función L-L. El método y función no influyen significativamente en el signo y forma de la curva de predicción.

A menos que se requiera de una alta precisión, la función L-L es muy útil para predecir el volumen de exceso molar de mezclas binarias de líquido iónico de imidazolio con solventes moleculares, ya que el procedimiento para medir índices de refracción de líquidos es más simple y en muchos casos, más económico que los métodos para determinar densidades.

La función A-B no predice adecuadamente el volumen de exceso molar desde índices de refracción en mezclas binarias liquido iónico de imidazolio con solvente molecular. Un análisis de la función indica que ésta considera las mezclas como soluciones ideales, lo cual contrasta con los valores de volumen de exceso molar reportados en la literatura para mezclas liquido iónicosolvente molecular.

\section{REFERENCIAS BIBLIOGRÁFICAS}

[1] Earle M.; and Seddon K. (2000) Ionic liquids. Green solvents for the future, En: Pure Appl. Chem., Vol. 72 (7), pp. 1391-1398.

[2] Olivier H. (1999). Recent developments in the use of non-aqueous ionic liquids for twophase catalysis, En: J. Mol. Catal. A. Vol. 146, pp. 285-289.

[3] Olivier-Bourbigou H. and Magna L. (2002). Ionic liquids: perspectives for organic and catalytic reactions, En: J. Mol. Catal. A. Vol. 182-183, pp. 419-437.

[4] Zhu J.; Chen J.; Li C; and Fei W. (2007). Centrifugal extraction for separation of ethylbenzene and octane using 1-butyl-3-methylimidazolium hexafluorophosphate ionic liquid as extractant, En: Sep. Purif. Technol. Vol.56, pp. 237-240.

[5] Matkowska D.; Goldon A.; and Hofman T. (2010). Densities, Excess Volumes, Isobaric Expansivities, and Isothermal Compressibilities of the 1-Ethyl-3-methylimidazolium Ethylsulfate + Ethanol System at temperatures (283.15 to 343.15) K and Pressures from (0.1 to 35) MPa, En: J. Chem. Eng. Data. Vol. 55, pp. 685-693.

[6] Welton T. (1999). Room-Temperature Ionic Liquids. Solvents for Synthesis and Catalysis. En: Chem. Rev. Vol. 99, pp. 2071-2083.

[7] Alonso L.; Arce A.; Francisco M.; and Soto A. (2008). Phase behaviour of 1 methyl-3octylimidazolium bis[trifluoromethylsulfonyl]imide with thiophene and aliphatic hydrocarbons: The influence of n-alkane chain length. En: Fluid Phase Equilib. Vol. 263, pp. 176-181. 
[8] Anantharaj R.; and Banerjee T. (2011). COSMO-RS based predictions for the desulphurization of diesel oil using ionic liquids: Effect of cation and anion combination. En: Fuel Proc. Tech. Vol. 92, pp. 39-52.

[9] Herbert M.; Galindo A.; and Montilla F. (2007). Catalytic epoxidation of cyclooctene using molybdenum(VI) compounds and urea-hydrogen peroxide in the ionic liquid [bmim]PF6. En: Catal. Commun. Vol. 8, pp. 987-990.

[10] Chen P. (2007). The assessment of removing strontium and cesium cations from aqueous solutions based on the combined methods of ionic liquid extraction and electrodeposition. En: Electrochim. Acta. Vol. 52, pp. 5484-5492.

[11] Berthod A.; Ruiz-Angel M.; and Carda-Broch, S. (2008). Ionic liquids in separation techniques. En: J. Chromatogr. A. Vol. 1184, pp. 6-18.

[12] Heintz, A. (2005). Recent developments in thermodynamics and thermophysics of nonaqueous mixtures containing ionic liquids. A review. En: J. Chem. Thermodynamics. Vol. 37, pp. 525-535.

[13] Zhu A.; Wang J.; and Liu R. (2010). A volumetric and viscosity study for the binary mixtures of 1-hexyl-3-methylimidazolium tetrafluoroborate with some molecular solvents. En: J. Chem. Thermodynamics., doi:10.1016/j.jct.2010.12.027.

[14] Kurnia K.; Taib M.; Mutalib M.; and Murugesan T. (2011). Densities, Refractive Indices and Excess Molar Volumes for Binary Mixtures of Protic Ionic Liquids with Methanol at $\mathrm{T}=$ (293.15 to 313.15) K. En: J. Mol. Liq., doi:10.1016/j.molliq. 2011.01.005.

[15] Shekaari H.; Mansoori Y.; and Sadeghi R., (2008). Density, speed of sound, and electrical conductance of ionic liquid 1-hexyl-3-methyl-imidazolium bromide in water at different temperatures. En: J. Chem. Thermodynamics. Vol. 40, pp. 852-859.

[16] Wang J.; Jiang H.; Liu Y.; and Hua Y. (2010). Density and surface tension of pure 1-ethyl3-methylimidazolium L-lactate ionic liquid and its binary mixtures with water. En: J. Chem. Thermodynamics., doi:10.1016/j.jct.2010.12.026.

[17] Domanska U. (2006). Thermophysical properties and thermodynamic phase behavior of ionic liquids. En: Thermochim. Acta. Vol. 448, pp. 19-30.

[18] Aralaguppi M., Jadar C., and Aminabhavi T. (1999). Density, Refractive Index, Viscosity, and Speed of Sound in Binary Mixtures of Cyclohexanone with Hexane, Heptane, Octane, Nonane, Decane, Dodecane, and 2,2,4-Trimethylpentane. En: J. Chem. Eng. Data. Vol. 44, pp. 435-440.

[19] Radovic I., Kijevcanin M., Gabrijel M., Serbanovic S., and Djordjevi B. (2008). Prediction of excess molar volumes of binary mixtures of organic compounds from refractive indices. En: Chemical Papers. Vol. 62 (3), pp. 302-312. 
[20] Piñeiro Á., Brocos P., Amigo A., Pintos M., and Bravo R. (1999). Surface tensions and refractive indices of (tetrahydrofuran $+n$-alkanes) at $T=298.15 \mathrm{~K}$. En: J. Chem. Thermodynamics. Vol. 31, pp. 931-942.

[21] Nakata M., and Sakurai M. (1987). Refractive index and excess volume for binary liquid mixtures. Part 1. Analyses of new and old data for binary mixtures. En: Journal of the Chemical Society, Faraday Transactions 1. Vol. 83, pp. 2449-2457.

[22] Heller W. (1965). Remarks on refractive index mixture rules. En: The Journal of Physical Chemistry. Vol. 69, pp. 1123-1129.

[23] Pereiro A.; and Rodriguez A. (2007). Study on the phase behaviour and thermodynamic properties of ionic liquids containing imidazolium cation with ethanol at several temperatures. En: J. Chem. Thermodynamics. Vol. 39, pp. 978-989.

[24] Pereiro A.; and Rodríguez A. (2007). Thermodynamic Properties of Ionic Liquids in Organic Solvents from (293.15 to 303.15) K. En: J. Chem. Eng. Data. Vol. 52, pp. 600-608.

[25] Pereiro A.; Tojo E.; Rodriguez A.; Canosa J.; and Tojo J. (2006). Properties of ionic liquid HMIMPF6 with carbonates, ketones and alkyl acetates. En: J. Chem. Thermodynamics. Vol. 38, pp. 651-661.

[26] Pereiro A.; and Rodriguez A. (2008). Measurement and correlation of (liquid + liquid) equilibrium of the azeotrope (cyclohexane +2 -butanone) with different ionic liquids at $T$ $=298.15 \mathrm{~K}$. En: J. Chem. Thermodynamics. Vol. 40, pp. 1282-1289.

[27] González E.; González B.; Calvar N.; and Domínguez A. (2007). Physical Properties of Binary Mixtures of the Ionic Liquid 1-Ethyl-3 methylimidazolium Ethyl Sulfate with Several Alcohols at $T$ ) $(298.15,313.15$, and 328.15) K and Atmospheric Pressure. En: J. Chem. Eng. Data. Vol. 52, pp. 1641-1646.

[28] Gómez E.; González B.; Calvar N.; Tojo E.; and Domínguez A. (2006). Physical Properties of Pure 1-Ethyl-3-methylimidazolium Ethylsulfate and Its Binary Mixtures with Ethanol and Water at Several Temperatures. En: J. Chem. Eng. Data. Vol. 51, pp. 2096-2102.

[29] Iglesias-Otero M.; Troncoso J.; Carballo E.; and Romaní L. (2008). Density and refractive index in mixtures of ionic liquids and organic solvents: Correlations and predictions. En: J. Chem. Thermodynamics. Vol. 40, pp. 949-956.

[30] Reis J.; Lampreia I.; Santos A.; Moita M.; and Douhéret G. (2010). Refractive Index of Liquid Mixtures: Theory and Experiment. En: ChemPhysChem. Vol. 11, pp. 3722-3733. 